\title{
Dynamic modeling and experimental verification of a cable-driven continuum manipulator with cable-constrained synchronous rotating mechanisms
}

\author{
Xudong Zheng - Taiwei Yang · Xianjin Zhu · Zhang Chen · Xueqian \\ Wang · Bin Liang
}

Received: date / Accepted: date

\begin{abstract}
The cable-driven segmented manipulator with cable-constrained synchronous rotating mechanisms (CCSRM) is a new type of continuum manipulator, which has large stiffness and less motors, and thus exhibits excellent comprehensive performance. This paper presents a dynamic modeling method for this type of manipulator to analyze the friction and deformation of the cables on the dynamical behaviors of the system. First, the driving cables are modeled based on the ALE formulation, the strategies for detecting stick-slip transitions are proposed by using a trial-and-error algorithm, and the stiff problems of the dynamic equations are released by a model smoothing method. Second, the dynamic modeling method for rigid links is presented by using quaternion parameters. Third, the connecting cables are modeled by torsional spring-dampers and the frictions between the connecting cables and the conduits are considered based on a modified Coulomb friction model. Finally, the numerical results are presented and verified by comparing with experiment results. The study shows that the friction and cable deforma-
\end{abstract}

X. Zheng · X. Wang

Tsinghua Shenzhen International Graduate School, Tsinghua

University, Shenzhen 518055, China

E-mail: xudongzheng_buaa@163.com

T. Yang

School of Mechanical Engineering and Automation, Harbin Institute of Technology, Shenzhen 518055, China

X. Zhu

School of Mechatronics Engineering, Harbin Institute of Technology, Harbin 150006, China

Z. Chen $(\bowtie) \cdot$ B. Liang

Department of Automation, Tsinghua University, Beijing 100084, China

E-mail: cz_da@tsinghua.edu.cn tion play an important role in the dynamical behaviors of the manipulator. Due to these two factors, the constant curvature bending of the segments does not remain.

Keywords Multibody dynamics · Cable-driven manipulator · ALE formulation · Stick-slip friction . Model smoothing method

\section{Introduction}

Nowadays, the cable-driven continuum manipulators have attracted the attentions of many researchers. Inspired by natural biology like octopus tentacles and elephan$\mathrm{t}$ trunks, continuum manipulators have high numbers of degree of freedoms (DOFs) and slim bodies, which enable them to work in confined spaces for dexterous operations. Cable-driven continuum manipulators have already been applied in many fields such as nuclear $[3$, medical application [4, and gas turbine engines [5]. Rone et al. 25] proposed a dynamic modeling method to capture curvature variations along a segment of manipulator by using a finite set of kinematic variables. Roesthuis and Misra 24 proposed a model to approximate the shape of a continuum manipulator by a serial chain of rigid links connected by flexible rotational joints. Qi et al. 23] presented a kinematic control method for continuum manipulators based on a fuzzy model-based approach. Falkenhahn et al. [6] developed a dynamic controller for continuum manipulators and achieved a good and intuitive dynamic behavior. Bajo and Simaan [1] studied the modeling, sensing, and control of multi-backbone continuum manipulator based on hybrid motion/force control method. Lakhal et al. [12] investigated a methodology for real-time solving of a 
complex kinematics of a class of continuum manipulators. Liu et al. [17] established an efficient dynamic model for a cable-driven continuum manipulator based on the principle of virtual power. Yuan et al. 35 presented a comprehensive static model for cable-driven multi-section continuum manipulators. Yang et al. 33. established a virtual work-based static model to study the deformation of a continuum robot with a generic rod-driven structure. Li et al. 14] compared three designs of continuum manipulator at the mechanism level from the kinematics point of view. Aiming at on-orbit services, Walker's group 28] proposed a new long and thin continuum manipulator; Jing et al. 9 presented a review on the kinematic analysis and dynamic stable control of continuum space manipulators; Huang et al. 8] studied the statics of continuum space manipulators with nonconstant curvature based on a pseudorigidbody 3R model; $\mathrm{Xu}$ et al. 31 proposed a modified modal method to solve the mission-oriented inverse kinematics of continuum space manipulators.

The above-mentioned continuum manipulators can mainly be divided into two categories: the first type is called a fully-driven rigid continuum manipulator, which is made up of multiple rigid links with universal joints, and each joint is driven by three cables (Fig. 1 (a)); the second type is named as a segmented elastic continuum manipulator, which consists of multiple segments of elastic backbone, while each segment is driven by three cables, too (Fig. 1 (b)). Each type has advantages and disadvantages: the first type can provide large stiffness, but it needs a large number of motors; on the contrary, the second type needs less motors, but it can hardly provide large stiffnessRN83. Aware of this, Xu's group [15,16] proposed a new type of cable-driven continuum manipulator with cable-constrained synchronous rotating mechanisms. As shown in Fig. 2, a segment of CCSRM is made up of several rigid links interconnected by universal joints, while each joint is connected with the adjacent joints by connecting cables, which enable the joints to rotate synchronously. The CCSRM is a new mechanical structure, and it can greatly reduce the number of motors as well as improve the stiffness of the manipulator. Li et al. [13] analyzed the friction and elastic deformation of the connecting cables on the CCSRM dynamical characteristics. Peng et al. 20, 19] proposed a dynamic modeling and trajectory tracking control method for this type of continuum manipulator. Ma et al. [18] proposed a dynamic model for this manipulator based on the methods of multibody system dynamics. However, to the authors' knowledge, there is no public literature which systematically deals with the effects of friction as well as deformation of driving and connecting cables on the dynamic behaviors of this type of manipulator. In real mechanical systems, these two factors cannot be ignored [40, 39, 30, 27, they may affect the motion accuracy of this kind of manipulator and restrict its further development.
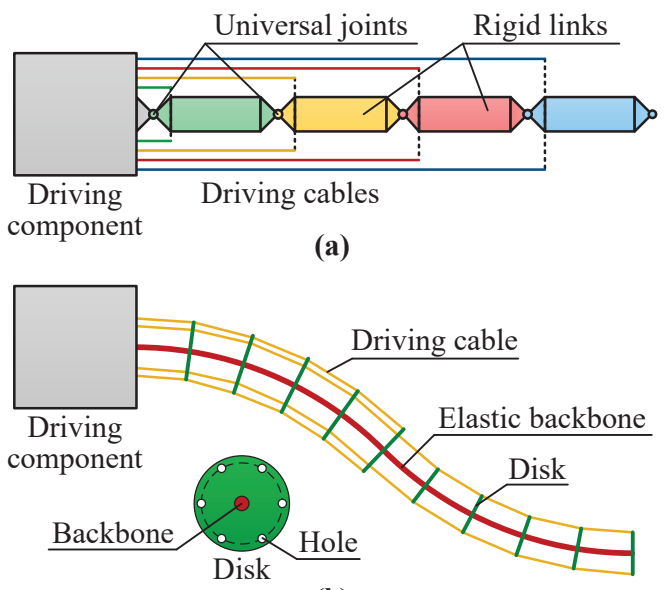

(b)

Fig. 1 Two types of cable-driven continuum manipulators: (a) Fully-driven rigid continuum manipulator; (b) Segmented elastic continuum manipulator

The difficulties for dynamic modeling of the cabledriven continuum manipulator with CCSRM include three aspects. First, this type of manipulator consists of a great number of cables. Traditional contact detection methods for cables 34 introduce hundreds of thousands of DOFs, which are difficult to solve. Recently, Peng et al. 21 proposed an arbitrary Lagrangian Eulerian (ALE) formulation for dynamic modeling of cabledriven mechanisms, which can significantly reduce the number of DOFs. However, if all cables, including driving and connecting cables, are modeled by ALE formulation, a great quantity of DOFs still be needed for this manipulator. Second, there are a lot of frictional contact points between the cables and rigid links, and the stick-slip events of the contact points should be detected during the solving procedure of dynamic equations 11,10]. Third, due to the high stiffness of cables, the dynamic equations of the manipulator are stiff, thus, they are often addressed by implicit solvers (stiff solvers) 36, 26]. However, the implicit solvers may have difficulties in the stick-slip transition points.

This paper presents a dynamic modeling method for the cable-driven continuum manipulator with CCSRM considering friction as well as deformation of all cables. Several methods are proposed to deal with the above three difficulties. First, the ALE method and a torsional spring-damper model are applied to significantly reduce the numbers of DOFs introduced by dynamic modeling of cables. Second, a trial-and-error strategy for detect- 


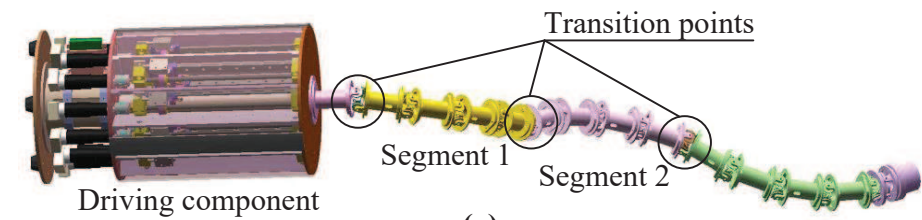

(a)

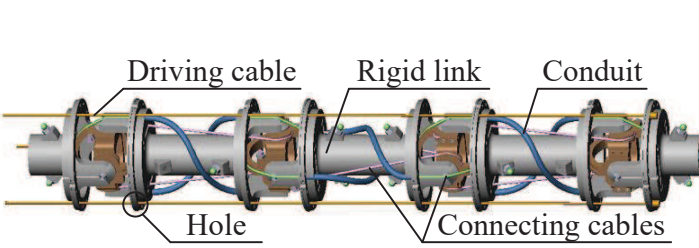

(b)

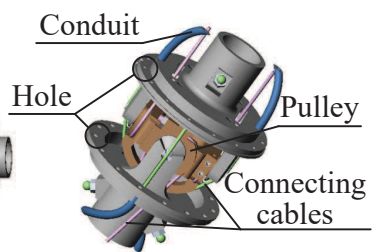

(c)

Fig. 2 (a) Cable-driven segmented continuum manipulator; (b) Cable-constrained synchronous rotating mechanism (CCSRM); (c) Universal joint

ing the stick-slip transitions of the driving cables and the holes is proposed. Third, the stiff problems are released by a model smoothing method [37, after which the dynamic equations can be solved by explicit solvers without difficulties in stick-slip transition points. The rest of this paper is organized as follows: in section 2 , the dynamic modeling method for driving cables is presented based on the ALE formulation and the strategies for detecting the stick-slip transitions are proposed; in section 3, the dynamic modeling method for the rigid links are given by using the quaternion parameters; in section 4, the connecting cables of the manipulator are modeled by torsional spring-dampers; in section 5 , the solving procedure for the dynamic equations is presented; in section 5 , the results are presented and verified by comparing with experiments; finally, in the last section, the main conclusions from this paper are drawn.

\section{Dynamic modeling of the driving cables via ALE formulation}

The cable-driven continuum manipulator studied in this paper is illustrated in Fig. 3. The manipulator consist$\mathrm{s}$ of a chain of rigid links interconnected by universal joints. The links and joints are labeled sequentially. The rigid links are divided into several segments, and in each segment the universal joints are connected one by one through connecting cables, which makes them rotate synchronously. Each segment of rigid links is driven by three driving cables, and the driving component is fixed on the ground. In order to obtain the dynamic model of the continuum manipulator, it is divided into three parts: the driving cables, the rigid links, and the connecting cables, which are described in detail in the following sections.

\subsection{Dynamic equations of an ALE element}

The ALE formulation has the advantage of sufficiently reducing the number of DOFs introduced by dynamic modeling of the cables, so it is introduced to model the driving cables. As shown in Fig. 4, the vector of generalized coordinates of an ALE cable element is expressed as

$\boldsymbol{q}_{\text {ele }}=\left[\boldsymbol{r}_{1}^{\mathrm{T}}, \boldsymbol{r}_{2}^{\mathrm{T}}, s_{1}, s_{2}\right]^{\mathrm{T}}$,

where $\boldsymbol{r}_{k}=\left[x_{k}, y_{k}, z_{k}\right]^{\mathrm{T}}(k=1,2)$ is the vector of global positions of node $k$ and $s_{k}$ is the material coordinate of the corresponding node.

An ALE node can be degenerated into a Lagrangian node or an Eulerian node by introducing some special constraints. A Lagragian node owns a constant material coordinate, but its position can move, i.e., $s_{k}-s_{0}=0$. On the contrary, an Eulerian node is fixed on the rigid links or ground, but its material coordinate can vary, i.e., $\boldsymbol{r}_{k}-\boldsymbol{r}_{0}=\mathbf{0}$.

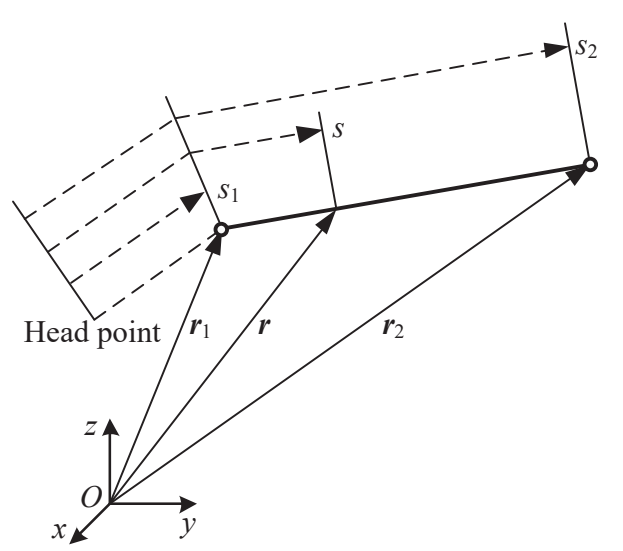

Fig. 4 Generalized coordinates of an ALE cable element 


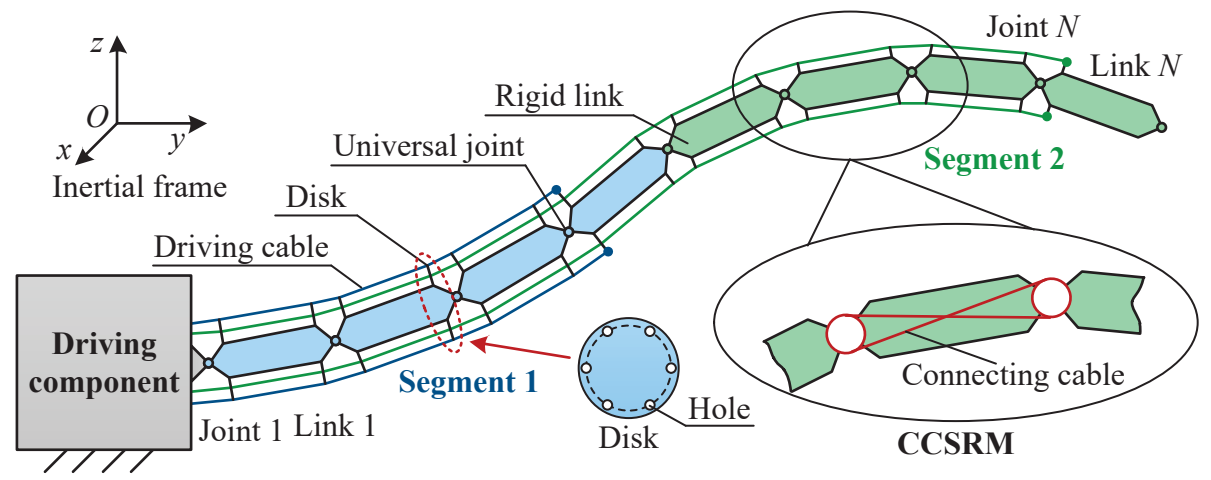

Fig. 3 Cable-driven continuum manipulator with cable-constrained synchronous rotating mechanisms (CCSRM)

The position vector of an arbitrary point in the element can be written as

$\boldsymbol{r}=\boldsymbol{N}_{r} \boldsymbol{q}_{r}$

where

$\left\{\begin{array}{l}\boldsymbol{q}_{r}=\left[\boldsymbol{r}_{1}^{\mathrm{T}}, \boldsymbol{r}_{2}^{\mathrm{T}}\right]^{\mathrm{T}}, \boldsymbol{N}_{r}=\left[N_{1} \boldsymbol{I}_{3}, N_{2} \boldsymbol{I}_{3}\right], \\ N_{1}=(1-\zeta) / 2, N_{2}=(1+\zeta) / 2, \\ \zeta=\frac{2 s-s_{1}-s_{2}}{s_{2}-s_{1}},\end{array}\right.$

in which $N_{1}$ and $N_{2}$ are shape functions and $\zeta$ is the local nature coordinate of the arbitrary point. Taking the first and second derivatives of Eq. (2) with respect to time yields

$$
\left\{\begin{aligned}
\dot{\boldsymbol{r}}= & \boldsymbol{N} \dot{\boldsymbol{q}}_{\text {ele }}, \ddot{\boldsymbol{r}}=\mathbf{N} \ddot{\boldsymbol{q}}_{\text {ele }}+\ddot{\boldsymbol{r}}_{s}, \\
\boldsymbol{N}= & {\left[\boldsymbol{N}_{r}, \frac{\partial \boldsymbol{N}_{r}}{\partial s_{1}} \boldsymbol{q}_{r}, \frac{\partial \boldsymbol{N}_{r}}{\partial s_{2}} \boldsymbol{q}_{r}\right], } \\
\ddot{\boldsymbol{r}}_{s}= & 2\left(\frac{\partial \boldsymbol{N}_{r}}{\partial s_{1}} \dot{s}_{1}+\frac{\partial \boldsymbol{N}_{r}}{\partial s_{2}} \dot{s}_{2}\right) \dot{\boldsymbol{q}}_{r} \\
& +\left(\frac{\partial^{2} \boldsymbol{N}_{r}}{\partial s_{1}^{2}} \dot{s}_{1}^{2}+2 \frac{\partial^{2} \boldsymbol{N}_{r}}{\partial s_{1} \partial s_{2}} \dot{s}_{1} \dot{s}_{2}+\frac{\partial^{2} \boldsymbol{N}_{r}}{\partial s_{2}^{2}} \dot{s}_{2}^{2}\right) \boldsymbol{q}_{r},
\end{aligned}\right.
$$

where the additional acceleration $\ddot{\boldsymbol{r}}_{s}$ results from the change of the material coordinates.

The dynamic equations of the ALE cable element can be obtained as 21

$\boldsymbol{M}_{\text {ele }} \ddot{\boldsymbol{q}}_{\text {ele }}+\boldsymbol{Q}_{f}+\boldsymbol{Q}_{e}+\boldsymbol{Q}_{p}=\mathbf{0}$,

where

$$
\left\{\begin{array}{l}
\boldsymbol{M}_{\text {ele }}=\frac{s_{2}-s_{1}}{2} \int_{-1}^{1} \rho A \boldsymbol{N}^{\mathrm{T}} \boldsymbol{N} \mathrm{d} \varsigma, \\
\boldsymbol{Q}_{f}=\frac{s_{2}-s_{1}}{2} \int_{-1}^{1} \boldsymbol{N}^{\mathrm{T}} \boldsymbol{f} \mathrm{d} \varsigma, \\
\boldsymbol{Q}_{e}=\frac{s_{2}-s_{1}}{2} \int_{-1}^{1}\left(\left(\frac{\partial \varepsilon}{\partial \boldsymbol{q}_{e l e}}\right)^{\mathrm{T}} E A(k \varepsilon+\beta \dot{\varepsilon})\right) \mathrm{d} \varsigma, \\
\boldsymbol{Q}_{p}=\frac{s_{2}-s_{1}}{2} \int_{-1}^{1} \rho A \boldsymbol{N}^{\mathrm{T}} \ddot{\boldsymbol{r}}_{s} \mathrm{~d} \varsigma, \\
\varepsilon=\frac{1}{2}\left(\frac{\partial \boldsymbol{r}^{\mathrm{T}}}{\partial s} \frac{\partial \boldsymbol{r}}{\partial s}-1\right), \\
k= \begin{cases}1, & \text { if } \varepsilon>0, \\
0, & \text { otherwise, }\end{cases}
\end{array}\right.
$$

in which $\boldsymbol{M}_{\text {ele }}$ is the generalized mass matrix, $\boldsymbol{Q}_{f}$ is the vector of generalized external forces including friction, $\boldsymbol{Q}_{e}$ is the vector of generalized elastic forces, $\boldsymbol{Q}_{p}$ is the vector of additional inertial forces, $\boldsymbol{f}$ is the vector of external forces, $E$ is the Young's modulus, $A$ is the cross sectional area, $\rho$ is the density of the cable, $\varepsilon$ is the axial strain, $k$ represents the cable can only resist tension but not compression, and $\beta$ is the Rayleigh damping factor.

\subsection{Frictional forces between the driving cables and} holes

As the thickness of the disk is small enough compared with element length, the hole can be viewed as an Eulerian node, while the cable between two adjacent holes can be meshed as an ALE cable element. Thus, the following constraint equation of Eulerian node $i$ is obtained:

$\boldsymbol{r}_{i}-\boldsymbol{r}_{\text {hole }, i}=\mathbf{0}$.

The Coulomb dry friction model is able to simulate static friction and to capture stick-slip motions, thus, it is adopted to describe the frictional forces acting at Eulerian nodes. The Coulomb dry friction model for point contact problems can be written as

$f= \begin{cases}-\mu N \operatorname{sgn}\left(v_{\tau}\right), & v_{\tau} \neq 0, \\ -\min \left(\left|F_{\tau}\right|, \mu^{\prime} N\right) \operatorname{sgn}\left(F_{\tau}\right), & v_{\tau}=0,\end{cases}$

where $f$ and $N$ are frictional force and normal contact force, respectively, $\mu$ and $\mu^{\prime}$ are kinetic and static friction coefficients, respectively, $v_{\tau}$ is the relative tangential velocity, and $F_{\tau}$ is the resultant of forces acting at the point in tangential direction. In numerical simulations, when $\left|v_{\tau}\right|$ is smaller than a tiny tolerance $v_{\varepsilon}$, it is viewed as zero, as shown in Fig. 5.

As depicted in Fig. 6, the frictional force acting on the cable can be derived from Eq. (8) as

$f_{t}= \begin{cases}T_{1}\left(1-e^{\mu \theta \operatorname{sgn}\left(v_{\tau}\right)}\right), & v_{\tau} \neq 0 \\ \min \left(\left|T_{1}-T_{2}\right|, f_{\max }\right) \operatorname{sgn}\left(T_{1}-T_{2}\right), & v_{\tau}=0\end{cases}$ 


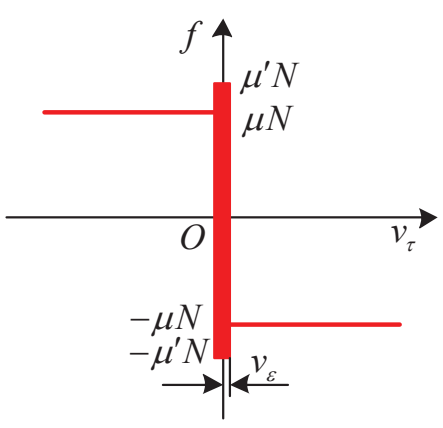

Fig. 5 Coulomb dry friction model

where $\theta$ is the wrap angle and $f_{\max }$ is the limiting static frictional force, and

$f_{\max }=\min \left(T_{1}, T_{2}\right)\left(e^{\mu^{\prime} \theta}-1\right)$,

which means the limiting static frictional force is determined by the smaller side of tension. It has been proved that the frictional force acts only on the material coordinates, and it is independent of the global positions 21. Thus, the vector of generalized forces acting at the Eulerian node can be written as

$\boldsymbol{Q}_{\text {friction }}=\left[\mathbf{0}_{1 \times 3}, f_{t}\right]^{\mathrm{T}}$.

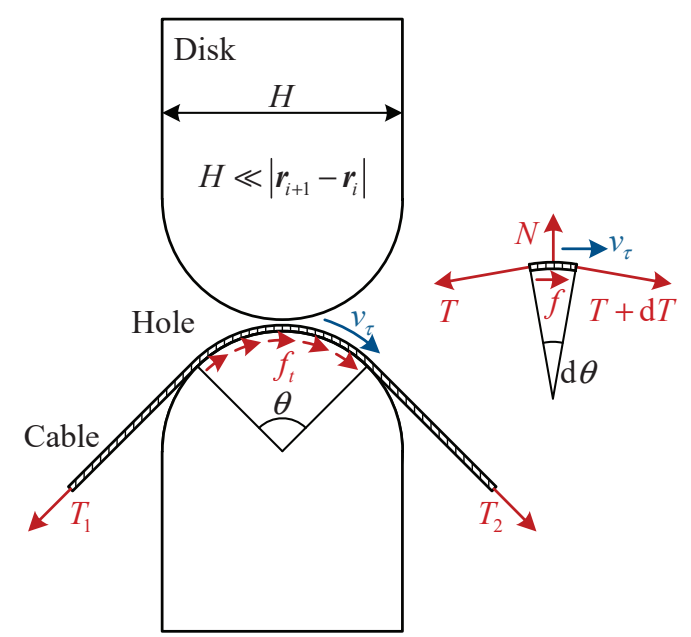

Fig. 6 Frictional force acting at the Eulerian node

\subsection{Strategies for detecting stick-slip motions}

The difficulty yielded by the Coulomb dry friction model is that it is hard to determine stick-slip states and to calculate static frictional forces during the transitions of the contact points from slipping to sticking or reversed slipping 38, 29. Fortunately, by using ALE formulation, the wrap angles, the relative tangential velocities, and the tensions of the cable elements can be determined, thus the stick-slip transitions and the frictional forces acting at the Eulerian nodes can be solved independently via trial-and-error algorithm.

As shown in Fig. 7, the wrap angles of node $i$ can be calculated as

$\theta_{i}=\pi-\arccos \left(\frac{\left(\boldsymbol{r}_{i-1}-\boldsymbol{r}_{i}\right) \cdot\left(\boldsymbol{r}_{i+1}-\boldsymbol{r}_{i}\right)}{\left|\boldsymbol{r}_{i-1}-\boldsymbol{r}_{i}\right|\left|\boldsymbol{r}_{i+1}-\boldsymbol{r}_{i}\right|}\right)$.

The tangential velocity of the cable relative to the hole in node $i$ is the negative of the derivative of material coordinate, i.e., $v_{\tau i}=-\dot{s}_{i}$. The tension of element $i$ can be solved as

$T_{i}=E A\left(k_{i} \varepsilon_{i}+\beta \dot{\varepsilon}_{i}\right)$,

where $\varepsilon_{i}$ is the axial strain of element $i$, which is determined by Eq. (6). After obtaining the above variables, the frictional forces acting at different Eulerian nodes can be calculated by Eq. (9) independently.

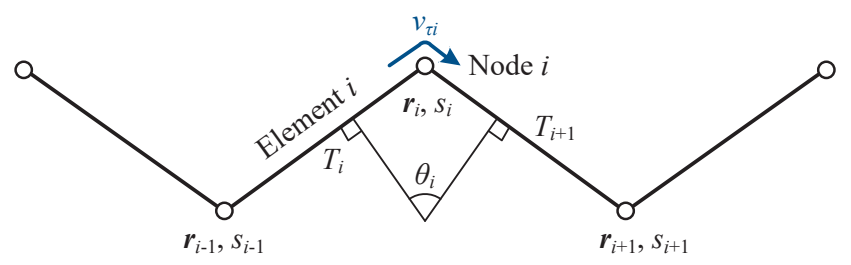

Fig. 7 The geometric relations of the adjacent nodes

2.4 Model smoothing method for modification of the dynamic equations of the ALE cable elements

Due to the high stiffness of the driving cables, the dynamic equations of the system are stiff, and thus they are often solved by implicit solvers, such as backward differentiation formula (BDF), implicit Runge-Kutta methods (Radau IIa). However, the implicit solvers need to calculate the Jacobian matrix of dynamic equations with respect to generalized coordinates and velocities, which is related to the partial derivative of frictional force with respect to relative tangential velocity. As shown in Fig. 5, in the transition points from slipping to sticking and reversed slipping, the partial derivative is not existent, so the implicit solvers may have trouble solving dynamic equations with stick-slip friction. To avoid the calculation of the above Jacobian matrix, combining with model smoothing method proposed by Ref. [37, the explicit solver can be applied. 
Since high frequency elastic vibrations in the system arise from the strains which change fast over time, therefore, in a time interval $(t, t+h)$, strain at time $\tau$ can be approximated to

$\varepsilon_{\tau}=\varepsilon_{t}+(\tau-t) \dot{\varepsilon}_{t}+\frac{1}{2}(\tau-t)^{2} \ddot{\varepsilon}_{t}$.

The time-averaged strain in this short time interval can then be expressed as

$\bar{\varepsilon} \triangleq \frac{1}{h} \int_{t}^{t+h} \varepsilon_{\tau} \mathrm{d} \tau=\varepsilon_{t}+\frac{h}{2} \dot{\varepsilon}_{t}+\frac{h^{2}}{6} \ddot{\varepsilon}_{t}$.

Similarly,

$\overline{\dot{\varepsilon}} \triangleq \frac{1}{h} \int_{t}^{t+h} \dot{\varepsilon}_{\tau} \mathrm{d} \tau=\dot{\varepsilon}_{t}+\frac{h}{2} \ddot{\varepsilon}_{t}$,

where $h$ is the short time period, which is also called the model smoothing step.

The essential of model smoothing method is substituting $\varepsilon$ and $\dot{\varepsilon}$ in Eq. (6) by $\bar{\varepsilon}$ and $\overline{\dot{\varepsilon}}$, which leads to

$\boldsymbol{Q}_{e}=\frac{s_{2}-s_{1}}{2} \int_{-1}^{1}\left(\left(\frac{\partial \varepsilon}{\partial \boldsymbol{q}_{\text {ele }}}\right)^{\mathrm{T}} E A\left(k \varepsilon+\beta^{\prime} \dot{\varepsilon}+\gamma \ddot{\varepsilon}\right)\right) \mathrm{d} \varsigma$,

where $\beta^{\prime}=\beta+\frac{k h}{2}, \gamma=\frac{\beta h}{2}+\frac{k h^{2}}{6}$, and the subscript $t$ is neglected.

As $\varepsilon=\varepsilon\left(\boldsymbol{q}_{\text {ele }}\right)$, the following equations can be generated:

$\left\{\begin{array}{l}\dot{\varepsilon}=\varepsilon_{\boldsymbol{q}_{\text {ele }}} \dot{\boldsymbol{q}}_{\text {ele }}, \\ \ddot{\varepsilon}=\dot{\varepsilon}_{\boldsymbol{q}_{\text {ele }}} \dot{\boldsymbol{q}}_{\text {ele }}+\varepsilon_{\boldsymbol{q}_{\text {ele }}} \ddot{\boldsymbol{q}}_{\text {ele }},\end{array}\right.$

where $\varepsilon_{\boldsymbol{q}_{\text {ele }}}=\partial \varepsilon / \partial \boldsymbol{q}_{\text {ele }}$ is a Jacobian matrix. Substituting Eq. (18) into Eqs. (17) and (5) leads to

$\left(\boldsymbol{M}_{\text {ele }}+\boldsymbol{M}_{\text {elastic }}\right) \ddot{\boldsymbol{q}}_{\text {ele }}+\boldsymbol{Q}_{f}+\boldsymbol{Q}^{\prime}{ }_{e}+\boldsymbol{Q}_{p}=\mathbf{0}$,

with

$\left\{\begin{array}{l}\boldsymbol{M}_{\text {elastic }}=\frac{s_{2}-s_{1}}{2} \int_{-1}^{1}\left(\varepsilon_{\boldsymbol{q}_{\text {ele }}}^{\mathrm{T}} E A \gamma \varepsilon_{\boldsymbol{q}_{\text {ele }}}\right) \mathrm{d} \varsigma, \\ \boldsymbol{Q}_{e}^{\prime}=\frac{s_{2}-s_{1}}{2} \int_{-1}^{1}\left(\varepsilon_{\boldsymbol{q}_{\text {ele }}}^{\mathrm{T}} E A\left(k \varepsilon+\beta^{\prime} \dot{\varepsilon}+\gamma \dot{\varepsilon}_{\boldsymbol{q}_{\text {ele }}} \dot{\boldsymbol{q}}_{\text {ele }}\right)\right) \mathrm{d} \varsigma .\end{array}\right.$

Compare Eqs. (5) and (19), it can be seen that additional inertia and damping terms are added to the dynamic equations of the ALE element. Thus, the natural frequency of the element is reduced accordingly, and the greater the parameter $h$, the less high frequency components retain. For general problems, the model smoothing step $h$ can be selected between $0.1 \mathrm{~s}$ and $0.001 \mathrm{~s}$.
It has been demonstrated that the model smoothing method can reduce high frequency component and amplitude, which we are usually not concerned about, and does not affect the rigid motion of the system (the modal with zero-order frequency) 37. The procedure of the method is remarkably concise and is straightforward to implement during the modeling process, which can be viewed as a new approach for the numerical solution of stiff problems.

\section{Dynamic modeling of the rigid links based on quaternion parameters}

\subsection{Kinematics of rigid links with quaternion parameters}

The quaternion parameters have the ability to avoid singularities as well as reduce the calculation of trigonometric functions to improve the calculation efficiency [32], thus they are adopted to describe the rotations of the rigid links. As illustrated in Fig. 8, the coordinate system $C_{i} x_{i}^{\prime} y_{i}^{\prime} z_{i}^{\prime}$ is a body-fixed reference frame on link $i$. The generalized coordinate vector of rigid link $i$ can be expressed as

$\boldsymbol{q}_{i}=\left[\boldsymbol{q}_{C i}^{\mathrm{T}}, \boldsymbol{q}_{e i}^{\mathrm{T}}\right]^{\mathrm{T}}$

where $\boldsymbol{q}_{C i}=\left[x_{i}, y_{i}, z_{i}\right]^{\mathrm{T}}$ is the generalized coordinate vector of the mass center of link $i$ and $\boldsymbol{q}_{e i}=\left[e_{0 i}, e_{1 i}, e_{2 i}, e_{3 i}\right]^{\mathrm{T}}$ is the vector of quaternion parameters. The global position vector of an arbitrary point A on the rigid link can be expressed as

$\boldsymbol{x}=\boldsymbol{q}_{C i}+\boldsymbol{R}_{i}^{\mathrm{T}} \boldsymbol{x}^{\prime}{ }_{C i}$,

in which $\boldsymbol{x}_{C i}^{\prime}$ is the position vector of arbitrary point A in the body-fixed reference frame and $\boldsymbol{R}_{i}$ is a rotation matrix.

It can be derived from Eq. (22) that the relative rotation matrix of link $i$ with respect to link $i-1$ is

$\boldsymbol{R}_{i /(i-1)}=\boldsymbol{R}_{i} \boldsymbol{R}_{i-1}^{\mathrm{T}}$,

where the subscript $i /(i-1)$ means link $i$ with respect to link $i-1$. Thus, the vector of relative rotation angles of link $i$ with respect to link $i-1$ can be determined as

$\boldsymbol{\theta}_{i /(i-1)}=\boldsymbol{f}_{\boldsymbol{R}-\boldsymbol{\theta}}\left(\boldsymbol{R}_{i /(i-1)}\right)$

in which the function of rotation matrix to Euler angles $\boldsymbol{f}_{\boldsymbol{R}-\boldsymbol{\theta}}$ is related to the rotation sequence. 


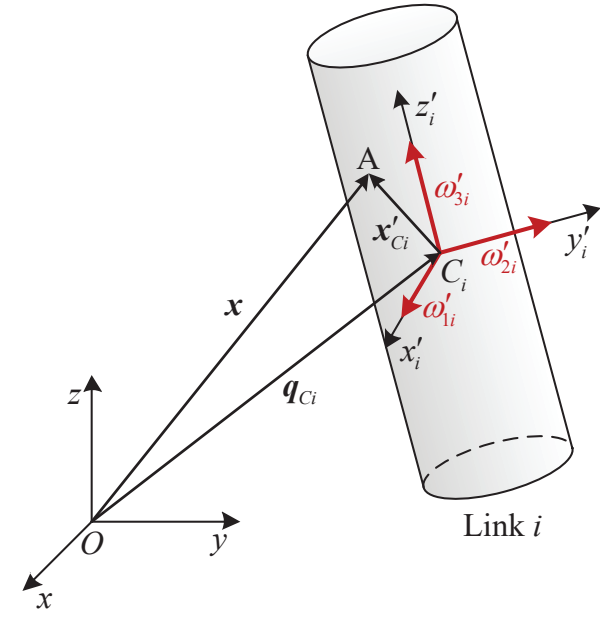

Fig. 8 Coordinates of a rigid link

The vector of angular velocities of link $i$ in the corresponding body-fixed reference frame is

$\boldsymbol{\omega}_{i}^{\prime}=\boldsymbol{G}_{i} \dot{\boldsymbol{q}}_{i}$,

where

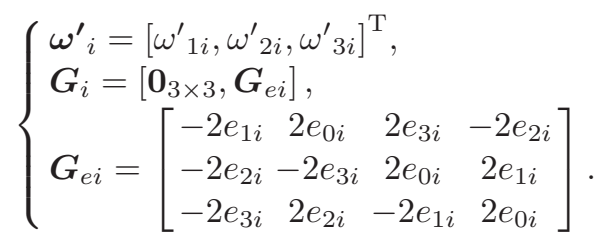

The vector of relative angular velocities of link $i$ with respect to link $i-1$ in the body-fixed reference frame of link $i$ is

$\boldsymbol{\omega}_{i /(i-1)}=\boldsymbol{\omega}_{i}^{\prime}-\boldsymbol{R}_{i /(i-1)} \boldsymbol{\omega}_{i-1}^{\prime}$.

According to Euler kinematical equations,

$\boldsymbol{\omega}_{i /(i-1)}=\boldsymbol{B}_{i /(i-1)} \dot{\boldsymbol{\theta}}_{i /(i-1)}$,

where the matrix $\boldsymbol{B}_{i /(i-1)}$ is determined by Euler rotation sequence and the vector of relative rotation angles $\boldsymbol{\theta}_{i /(i-1)}$. From Eq. (28), the following equation can be generated:

$\dot{\boldsymbol{\theta}}_{i /(i-1)}=\boldsymbol{B}_{i /(i-1)}^{-1} \boldsymbol{\omega}_{i /(i-1)}$.

\subsection{Dynamic equations of a rigid link}

The kinetic energy of the rigid link $i$ can be expressed as

$T_{i}=\frac{1}{2} \dot{\boldsymbol{q}}_{i}^{\mathrm{T}} \boldsymbol{m}_{i} \dot{\boldsymbol{q}}_{i}$ where the mass matrix $\boldsymbol{m}_{i}$ is

$\boldsymbol{m}_{i}=\left[\begin{array}{cc}m_{i} \boldsymbol{I}_{3} & \mathbf{0}_{3 \times 4} \\ \mathbf{0}_{4 \times 3} & \boldsymbol{G}_{e i}^{\mathrm{T}} \boldsymbol{J}_{i}^{\prime} \boldsymbol{G}_{e i}\end{array}\right]$

in which $m_{i}$ is the mass of link $i$ and $\boldsymbol{J}^{\prime}{ }_{i}$ is the inertia matrix about the corresponding body-fixed reference frame. The vector of generalized forces acting on rigid link $i$ is

$\boldsymbol{Q}_{i}=\left[\begin{array}{c}\boldsymbol{F}_{i} \\ \boldsymbol{G}_{e i}^{\mathrm{T}} \boldsymbol{M}^{\prime}{ }_{i}\end{array}\right]$

in which $\boldsymbol{F}_{i}$ and $\boldsymbol{M}^{\prime}{ }_{i}$ are the vectors of forces and moments, respectively, which include external applied forces and moments from the connecting cables, etc. Thus, the dynamic equations of a free rigid link can be obtained as

$\boldsymbol{m}_{i} \ddot{\boldsymbol{q}}_{i}+\tilde{\boldsymbol{Q}}_{i}=\mathbf{0}$

where

$\tilde{\boldsymbol{Q}}_{i}=\dot{\boldsymbol{m}}_{i} \dot{\boldsymbol{q}}_{i}-\frac{\partial T_{i}}{\partial \boldsymbol{q}_{i}}-\boldsymbol{Q}_{i}$

\subsection{Constraint equations of the rigid links}

The following three types of kinematic constraint equations of the rigid links will be used to obtain the dynamic equations of the whole multibody system. The first type of constraint is generated from the property of quaternions, that is

$g_{e i}=\boldsymbol{q}_{e i}^{\mathrm{T}} \boldsymbol{q}_{e i}-1=0$.

The second type is the position constraint in the universal joints, which means that the center points of a universal joint on two adjacent rigid links coincide, i.e.,

$\boldsymbol{g}_{p, i, i+1}=\boldsymbol{q}_{C i}+\boldsymbol{R}_{i}^{\mathrm{T}} \boldsymbol{x}_{C i}^{\prime}-\boldsymbol{q}_{C(i+1)}-\boldsymbol{R}_{i+1}^{\mathrm{T}} \boldsymbol{x}_{C(i+1)}=\mathbf{0}$.

The third type is the orientation constraint in the universal joints, namely,

$g_{o, i, i+1}=\left(\boldsymbol{R}_{i}^{\mathrm{T}} \boldsymbol{o}_{i}^{\prime}\right)^{\mathrm{T}} \boldsymbol{R}_{i+1}^{\mathrm{T}} \boldsymbol{o}_{i+1}^{\prime}=0$,

where $\boldsymbol{o}^{\prime}{ }_{i}$ and $\boldsymbol{o}^{\prime}{ }_{i+1}$ are unit vectors on rigid links $i$ and $i+1$, respectively. The geometric relationship in a universal joint is shown in Fig. 9. 


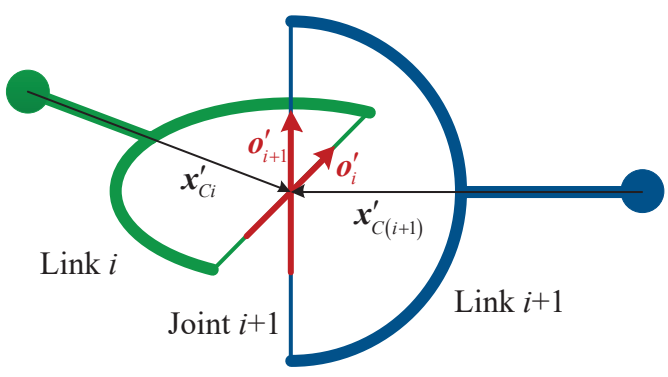

Fig. 9 The geometric relationship of a universal joint

\section{Dynamic modeling of the connecting cables}

As shown in Fig. 10, the cable-constrained synchronous rotating mechanisms contain two kinds, which are named as large "S" mechanisms and small "S" mechanisms. The types of friction acting on the connecting cables are different according to the kinds of synchronous rotating mechanisms. The connecting cables of small "S" mechanisms suffer from stick frictional forces of the pulleys, and the slip motions can be ignored. On the contrary, the connecting cables of large "S" mechanisms are subjected to slip or stick-slip frictional forces from the pulleys and conduits. Thus, the dynamic modeling of these two kinds of mechanism are different. In order to avoid further use of cable elements, which introduce the numbers of DOFs to the multibody system, the dynamic modeling of the connecting cables are different from the driving cables, as elaborated in the following part of this section.

4.1 Dynamic modeling of connecting cables for small "S" mechanisms

To ensure the connecting cables are always in tensioned states, they are applied by an initial pre-tensioning force. As illustrated in Fig. 11, consider two adjacent joints labeled as $i$ and $j(j=i+1)$ with connecting cables. If the joints rotate with certain angles, there will be a change in the strains of the cables as follows:

$\left\{\begin{array}{l}\varepsilon_{1}=\varepsilon_{0}-r_{s c}\left(\theta_{j}-\theta_{i}\right) / l_{s c}, \\ \varepsilon_{2}=\varepsilon_{0}+r_{s c}\left(\theta_{j}-\theta_{i}\right) / l_{s c},\end{array}\right.$

where $\varepsilon_{1}$ and $\varepsilon_{2}$ are strains of the couple of connecting cables, $\varepsilon_{0}$ is the initial pre-tensioning strain, $l_{s c}$ is the length of the cables, $r_{s c}$ is the radius of the pulleys, and $\theta_{i}$ and $\theta_{j}$ are the rotating angles of the two joints. The moment acting at joint $i$ from the connecting cables is

$M_{c i}=E_{c} A_{c} r_{s c}\left(\varepsilon_{2}-\varepsilon_{1}\right)$, in which $E_{c}$ and $A_{c}$ are the Young's modulus and crosssectional area of the connecting cables, respectively. Substituting Eq. (38) into Eq. (39) yields

$M_{c i}=k_{s c}\left(\theta_{j}-\theta_{i}\right)$,

where $k_{s c}$ is the connecting stiffness parameter, and

$k_{s c}=2 E_{c} A_{c} r_{s c}^{2} / l_{s c}$.

It can be seen from Eq. (41) that the connecting stiffness parameter of a small " $\mathrm{S}$ " mechanism is not related to the initial pre-tensioning force, and it retains constant as the universal joints rotate. If the damping effect of the connecting cables is considered, a damping term can be added to Eq. (40) as

$M_{c i}=k_{s c}\left(\theta_{j}-\theta_{i}\right)+c_{s c}\left(\dot{\theta}_{j}-\dot{\theta}_{i}\right)$,

where $c_{s c}$ is the connecting damping parameter. Similarly, the moment acting at joint $j$ from the connecting cables is written as

$M_{c j}=k_{s c}\left(\theta_{i}-\theta_{j}\right)+c_{s c}\left(\dot{\theta}_{i}-\dot{\theta}_{j}\right)$.

The physical meaning of Eqs. (42) and (43) is that the couple of connecting cables in the small "S" mechanism are modeled as a linear torsional spring-damper.

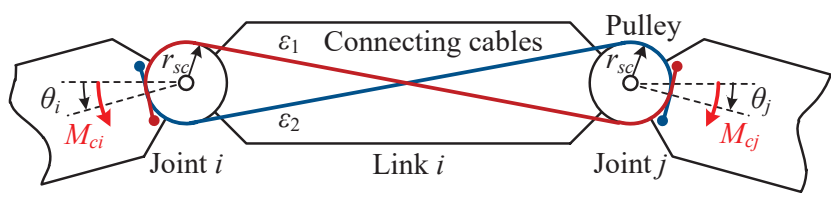

Fig. 11 Principle of a small "S" synchronous rotating mechanism

4.2 Dynamic modeling of connecting cables for large "S" mechanisms

The dynamic modeling of a large "S" synchronous rotating mechanism is much more complicated than that of a small "S" mechanism, since the connecting cables may exhibit stick-slip motions relative to the pulleys and conduits. For simplicity, a modified Coulomb fiction model is applied to describe the frictional forces acting on the connecting cables. The friction model is expressed as 22

$f=\mu_{m}\left(v_{\tau}\right) N$ 


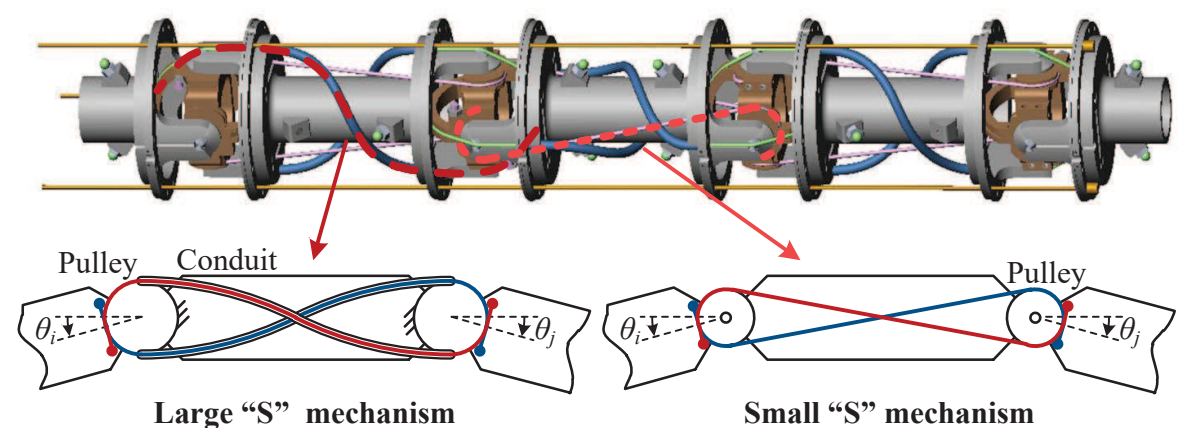

Fig. 10 Two kinds of cable-constrained synchronous rotating mechanisms

where

$$
\mu_{m}\left(v_{\tau}\right)=\left\{\begin{array}{cl}
-\mu_{c} \operatorname{sgn}\left(v_{\tau}\right), & \left|v_{\tau}\right|>v_{d}, \\
-\left\{\mu_{c}+\left(\mu^{\prime}{ }_{c}-\mu_{c}\right)\left(\frac{\left|v_{\tau}\right|-v_{s}}{v_{d}-v_{s}}\right)\right. & v_{s} \leq v_{\tau} \mid \leq v_{d}, \\
\left.\cdot\left[3-2\left(\frac{\left|v_{\tau}\right|-v_{s}}{v_{d}-v_{s}}\right)\right]\right\} \operatorname{sgn}\left(v_{\tau}\right), & \\
\mu^{\prime}{ }_{c}-2 \mu^{\prime}{ }_{c}\left(\frac{v_{\tau}+v_{s}}{2 v_{s}}\right)^{2}\left(3-\frac{v_{\tau}+v_{s}}{v_{s}}\right), & \left|v_{\tau}\right|<v_{s},
\end{array}\right.
$$

in which $v_{s}$ and $v_{d}$ are two velocity tolerances, $\mu_{c}$ and $\mu_{c}{ }^{\prime}$ are friction coefficients, and other variables are the same as Eq. (8). The graphic expression of this modified friction model is illustrated in Fig. 12. The friction model cannot capture stick state in a strict sense but allows a tiny slip velocity smaller than $v_{s}$ to produce an effect similar to static friction. Thus, this model avoids the detection of stick-slip states and simplifies the calculation of frictional forces.

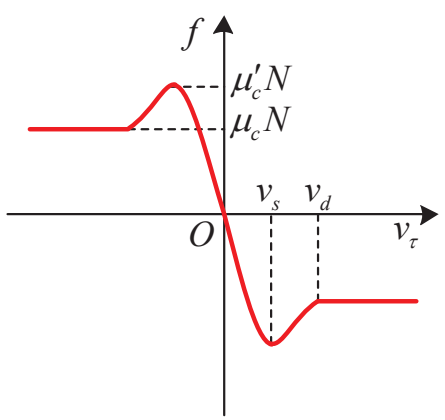

Fig. 12 A modified Coulomb friction model

As shown in Fig. 13, by adopting the modified Coulom$\mathrm{b}$ friction model, the tensions of the connecting cables in their two ends can be approximately derived as

$$
\left\{\begin{array}{c}
T_{i 1}=\frac{E_{c} A_{c} \mu_{m}\left(v_{\tau 1}\right) \Theta_{l c} \Delta l_{1}}{l_{l c}\left(1-e^{-\mu_{m}\left(v_{\tau 1}\right) \Theta_{l c}}\right)} \\
T_{j 1}=\frac{E_{c} A_{c} \mu_{m}\left(v_{\tau 1}\right) \Theta_{l c} \Delta l_{1} e^{-\mu_{m}\left(v_{\tau 1}\right) \Theta_{l c}}}{l_{l c}\left(1-e^{-\mu_{m}\left(v_{\tau 1}\right) \Theta_{l c}}\right)}
\end{array}\right.
$$

and

$$
\left\{\begin{array}{c}
T_{i 2}=\frac{E_{c} A_{c} \mu_{m}\left(v_{\tau 2}\right) \Theta_{l c} \Delta l_{2}}{l_{l c}\left(1-e^{-\mu_{m}\left(v_{\tau 2}\right) \Theta_{l c}}\right)} \\
T_{j 2}=\frac{E_{c} A_{c} \mu_{m}\left(v_{\tau 2}\right) \theta_{0} \Delta l_{2} e^{-\mu_{m}\left(v_{\tau 2}\right) \Theta_{l c}}}{l_{l c}\left(1-e^{-\mu_{m}\left(v_{\tau 2}\right) \Theta_{l c}}\right)}
\end{array}\right.
$$

where $l_{l c}$ is the length of large "S" connecting cables, $\Theta_{l c}$ is the special wrap angle, $v_{\tau 1}$ and $v_{\tau 2}$ are the tangential velocities of the connecting cables relative to the conduits, and $\Delta l_{1}$ and $\Delta l_{2}$ are the elongations of the couple of connecting cables, which are determined by

$\left\{\begin{array}{l}\Delta l_{1}=\frac{T_{p} l_{l c}}{E_{c} A_{c}}-r_{l c}\left(\theta_{j}-\theta_{i}\right) \\ \Delta l_{2}=\frac{T_{p} l_{l c}}{E_{c} A_{c}}+r_{l c}\left(\theta_{j}-\theta_{i}\right)\end{array}\right.$

in which $T_{p}$ is the pre-tensioning force and $r_{l c}$ is the radius of the pulley. Thus, the moment acting at joint $i$ from the connecting cables can be derived as

$M_{c i}=M_{p i}+k_{l c i}\left(\theta_{j}-\theta_{i}\right)$,

where

$\left\{\begin{array}{c}M_{p i}=T_{p} r_{l c}\left(\frac{\mu_{m}\left(v_{\tau 2}\right) \Theta_{l c}}{1-e^{-\mu_{m}\left(v_{\tau 2}\right) \Theta_{l c}}}-\frac{\mu_{m}\left(v_{\tau 1}\right) \Theta_{l c}}{1-e^{-\mu_{m}\left(v_{\tau 1}\right) \Theta_{l c}}}\right), \\ k_{l c i}=\frac{E_{c} A_{c} r_{l c}^{2}}{l_{l c}}\left(\frac{\mu_{m}\left(v_{\tau 2}\right) \Theta_{l c}}{1-e^{-\mu_{m}\left(v_{\tau 2}\right) \Theta_{l c}}}+\frac{\mu_{m}\left(v_{\tau 1}\right) \Theta_{l c}}{1-e^{-\mu_{m}\left(v_{\tau 1}\right) \Theta_{l c}}}\right) .\end{array}\right.$

By adding a damping term, the moment can be rewritten as

$M_{c i}=M_{p i}+k_{l c i}\left(\theta_{j}-\theta_{i}\right)+c_{l c i}\left(\dot{\theta}_{j}-\dot{\theta}_{i}\right)$.

Similarly, the moment acting at joint $j$ is expressed as

$M_{c j}=M_{p j}+k_{l c j}\left(\theta_{i}-\theta_{j}\right)+c_{l c j}\left(\dot{\theta}_{i}-\dot{\theta}_{j}\right)$, 
where

$$
\left\{\begin{aligned}
M_{p j} & =T_{p} r_{l c}\left(\frac{\mu_{m}\left(v_{\tau 1}\right) \Theta_{l c} e^{-\mu_{m}\left(v_{\tau 1}\right) \Theta_{l c}}}{1-e^{-\mu_{m}\left(v_{\tau 1}\right) \Theta_{l c}}}\right. \\
& \left.-\frac{\mu_{m}\left(v_{\tau 2}\right) \Theta_{l c} e^{-\mu_{m}\left(v_{\tau 2}\right) \Theta_{l c}}}{1-e^{-\mu_{m}\left(v_{\tau 2}\right) \Theta_{l c}}}\right) \\
k_{l c j}= & \frac{E_{c} A_{c} r_{l c}^{2}}{l_{l c}}\left(\frac{\mu_{m}\left(v_{\tau 1}\right) \Theta_{l c} e^{-\mu_{m}\left(v_{\tau 1}\right) \Theta_{l c}}}{1-e^{-\mu_{m}\left(v_{\tau 1}\right) \Theta_{l c}}}\right. \\
& \left.+\frac{\mu_{m}\left(v_{\tau 2}\right) \Theta_{l c} e^{-\mu_{m}\left(v_{\tau 2}\right) \Theta_{l c}}}{1-e^{-\mu_{m}\left(v_{\tau 2}\right) \Theta_{l c}}}\right)
\end{aligned}\right.
$$

which shows that by considering the friction acting on the connecting cables, the connecting stiffness parameters are no longer constant, and moments acting at the adjacent joints are related to the pre-tensional force of the connecting cables.

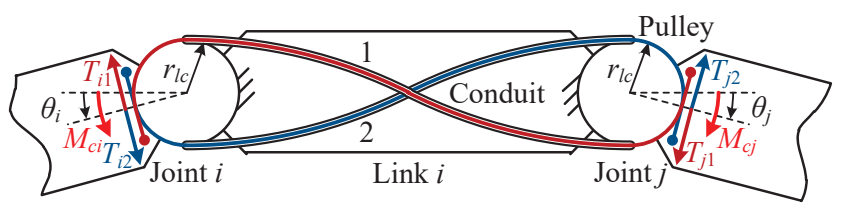

Fig. 13 Principle of a large "S" synchronous rotating mechanism

4.3 Vector of generalized forces generated from the connecting cables

It can be derived from Eqs. (42), (43), (51) and (52) that the vector of moments acting at joint $i$ from the nearby connecting cables is

$$
\begin{aligned}
\boldsymbol{M}_{\text {joint } i}= & \boldsymbol{M}_{p i}+\boldsymbol{k}_{c}\left(\boldsymbol{\theta}_{(i-1) /(i-2)}+\boldsymbol{\theta}_{(i+1) / i}-2 \boldsymbol{\theta}_{i /(i-1)}\right) \\
& +\boldsymbol{c}_{c}\left(\dot{\boldsymbol{\theta}}_{(i-1) /(i-2)}+\dot{\boldsymbol{\theta}}_{(i+1) / i}-2 \dot{\boldsymbol{\theta}}_{i /(i-1)}\right)
\end{aligned}
$$

where the matrices $\boldsymbol{k}_{c}$ and $\boldsymbol{c}_{c}$ contain the connecting stiffness and damping parameters, respectively. The above vector of moments acting on link $i$ in the body-fixed reference frame expression is

$\boldsymbol{M}_{c i}^{\prime}=\boldsymbol{B}_{i /(i-1)} \boldsymbol{M}_{\text {joint } i}$

while acting on link $i-1$ in the corresponding bodyfixed reference frame is

$\boldsymbol{M}_{c(i-1)}^{\prime}=-\boldsymbol{R}_{i /(i-1)}^{\mathrm{T}} \boldsymbol{B}_{i /(i-1)} \boldsymbol{M}_{\text {joint } i}$.

Then, the vector of generalized forces acting on rigid link $i$ yielded from the vector of moments $\boldsymbol{M}_{\text {joint } i}$ can be obtained as

$\boldsymbol{Q}_{c i}=\boldsymbol{G}_{i}^{\mathrm{T}} \boldsymbol{B}_{i /(i-1)} \boldsymbol{M}_{\text {joint } i}$.
Similarly,

$\boldsymbol{Q}_{c(i-1)}=-\boldsymbol{G}_{i-1}^{\mathrm{T}} \boldsymbol{R}_{i /(i-1)}^{\mathrm{T}} \boldsymbol{B}_{i /(i-1)} \boldsymbol{M}_{\text {joint } i}$.

\section{Dynamic equations of the whole system and solving procedure}

5.1 Dynamic equations of the whole multibody system

The vector of generalized coordinates of the whole manipulator system is

$\boldsymbol{q}=\left[\boldsymbol{r}_{1}^{\mathrm{T}}, s_{1}, \cdots, \boldsymbol{r}_{M}^{\mathrm{T}}, s_{M}, \boldsymbol{q}_{1}^{\mathrm{T}}, \cdots, \boldsymbol{q}_{N}^{\mathrm{T}}\right]^{\mathrm{T}}$,

where $M$ and $N$ are the numbers of driving cable nodes and rigid links, respectively. By assembling the mass matrices, generalized force vectors, and kinematic constraints of all bodies, the dynamic equations of whole manipulator system can be expressed in the following form:

$$
\left\{\begin{array}{c}
\boldsymbol{M}(\boldsymbol{q}, t) \ddot{\boldsymbol{q}}+\boldsymbol{Q}(\dot{\boldsymbol{q}}, \boldsymbol{q}, t)+\boldsymbol{\Phi}_{\boldsymbol{q}}^{\mathrm{T}} \boldsymbol{\lambda}=\mathbf{0} \\
\boldsymbol{\Phi}(\boldsymbol{q}, t)=\mathbf{0}
\end{array}\right.
$$

where $M$ is the mass matrix of whole system, which consists of mass matrices of the driving cables, rigid links, and the additional inertia terms yielded by model smoothing method in Eq. (20). $\boldsymbol{Q}$ is the vector of generalized forces including elastic forces, Coriolis forces, external applied forces, the frictional forces, and moments from the connecting cables. $\boldsymbol{\lambda}$ is the vector of Lagrange multipliers. $\boldsymbol{\Phi}$ is the vector of constraint equations, which includes the constraints from the Eulerian or Lagragian nodes and the rheonomic cable length input, the constraints of rigid links described in Section 3.3, and $\boldsymbol{\Phi}_{\boldsymbol{q}}=\partial \boldsymbol{\Phi} / \partial \boldsymbol{q}$.

5.2 Solving procedure for the cable-driven continuum manipulator with CCSRM

To restrain the drift of constraint violations, the Baumgarte stabilization method is introduced [2]

$\ddot{\boldsymbol{\Phi}}+\alpha_{B} \dot{\boldsymbol{\Phi}}+\beta_{B} \boldsymbol{\Phi}=\mathbf{0}$

where the positive parameters $\alpha_{B}$ and $\beta_{B}$ can be chosen following the instruction of Ref. [7]. Substituting $\dot{\boldsymbol{\Phi}}=$ $\boldsymbol{\Phi}_{\boldsymbol{q}} \dot{\boldsymbol{q}}+\partial \boldsymbol{\Phi} / \partial t$ into Eq. (61) yields

$\Phi_{q} \ddot{q}+b=0$, 
where

$$
\left\{\begin{array}{l}
\boldsymbol{b}=\dot{\boldsymbol{\Phi}}_{\boldsymbol{q}} \dot{\boldsymbol{q}}+\dot{\boldsymbol{\Phi}}_{t}+\alpha_{B} \boldsymbol{\Phi}_{\boldsymbol{q}} \dot{\boldsymbol{q}}+\alpha_{B} \boldsymbol{\Phi}_{t}+\beta_{B} \boldsymbol{\Phi} \\
\boldsymbol{\Phi}_{t}=\partial \boldsymbol{\Phi} / \partial t
\end{array}\right.
$$

Combining Eqs. (60) and (62) leads to

$$
\left(\boldsymbol{M}+\boldsymbol{\Phi}_{\boldsymbol{q}}^{\mathrm{T}} \boldsymbol{\Phi}_{\boldsymbol{q}}\right) \ddot{\boldsymbol{q}}+\boldsymbol{\Phi}_{\boldsymbol{q}}^{\mathrm{T}} \boldsymbol{b}+\boldsymbol{Q}+\boldsymbol{\Phi}_{\boldsymbol{q}}^{\mathrm{T}} \boldsymbol{\lambda}=\mathbf{0},
$$

The mass matrix $\boldsymbol{M}$ is singular, which is a property of ALE formulation. Added by $\boldsymbol{\Phi}_{\boldsymbol{q}}^{\mathrm{T}} \boldsymbol{\Phi}_{\boldsymbol{q}}$, it becomes invertible, thus,

$\ddot{\boldsymbol{q}}=-\left(\boldsymbol{M}+\boldsymbol{\Phi}_{\boldsymbol{q}}^{\mathrm{T}} \boldsymbol{\Phi}_{\boldsymbol{q}}\right)^{-1}\left(\boldsymbol{\Phi}_{\boldsymbol{q}}^{\mathrm{T}} \boldsymbol{b}+\boldsymbol{Q}+\boldsymbol{\Phi}_{\boldsymbol{q}}^{\mathrm{T}} \boldsymbol{\lambda}\right)$.

Substituting Eq. (65) into Eq. (62) yields the vector of Lagrange multipliers

$\boldsymbol{\lambda}=\boldsymbol{A}_{\lambda}^{-1} \boldsymbol{b}_{\lambda}$

with

$$
\left\{\begin{array}{l}
\boldsymbol{A}_{\lambda}=\boldsymbol{\Phi}_{\boldsymbol{q}}\left(\boldsymbol{M}+\boldsymbol{\Phi}_{\boldsymbol{q}}^{\mathrm{T}} \boldsymbol{\Phi}_{\boldsymbol{q}}\right)^{-1} \boldsymbol{\Phi}_{\boldsymbol{q}}^{\mathrm{T}}, \\
\boldsymbol{b}_{\lambda}=\boldsymbol{b}-\boldsymbol{\Phi}_{\boldsymbol{q}}\left(\boldsymbol{M}+\boldsymbol{\Phi}_{\boldsymbol{q}}^{\mathrm{T}} \boldsymbol{\Phi}_{\boldsymbol{q}}\right)^{-1}\left(\boldsymbol{\Phi}_{\boldsymbol{q}}^{\mathrm{T}} \boldsymbol{b}+\boldsymbol{Q}\right) .
\end{array}\right.
$$

Inserting Eq. (66) in Eq. (65), the dynamic equations can be transformed into the form of ODE as

$$
\ddot{\boldsymbol{q}}=-\left(\boldsymbol{M}+\boldsymbol{\Phi}_{\boldsymbol{q}}^{\mathrm{T}} \boldsymbol{\Phi}_{\boldsymbol{q}}\right)^{-1}\left(\boldsymbol{\Phi}_{\boldsymbol{q}}^{\mathrm{T}} \boldsymbol{b}+\boldsymbol{Q}+\boldsymbol{\Phi}_{\boldsymbol{q}}^{\mathrm{T}} \boldsymbol{A}_{\lambda}^{-1} \boldsymbol{b}_{\lambda}\right) .
$$

As the stiff problem is released by model smoothing method, the above equations can be solved efficiently by explicit ODE solvers. In this paper, the explicit Runge-Kutta method of fourth and fifth orders with variable step size (RK45 method) is applied, since it has advantages in both calculation accuracy and convergence [37.

\section{Results and discussion}

To verify the feasibility and correctness of the proposed dynamic modeling method, the numerical and experimental results of a cable-driven continuum manipulator are presented in this section. As shown in Fig. 14, the manipulator consists of two segments of CCSRM, each of which is made up of six rigid links. There are six driving cables in total, which are labeled in sequence. Cables 1, 2, and 3 drive the first segment, while cables 4,5 , and 6 drive the second segment. The positions of these driving cables are illustrated in the figure, and other important parameters of this manipulator are listed in Table 1. The gravitational acceleration is assumed to be zero in this part.
In order to exhibit the performance of cable-constrained synchronous rotating mechanisms both in simulations and experiments, the planned motion of the manipulator is that each joint rotates $6^{\circ}$ around $x_{i-1}^{\prime}$ axis, and then rotates back, as illustrated in Fig. 14. The input lengths of driving cables are determined by a pure kinematical planning method without cable deformations [20]. To test the stick-slip capturing capacity of our proposed simulation method, in seconds 20 to 24 , the input cable lengths remain unchanged, as shown in Fig. 15.

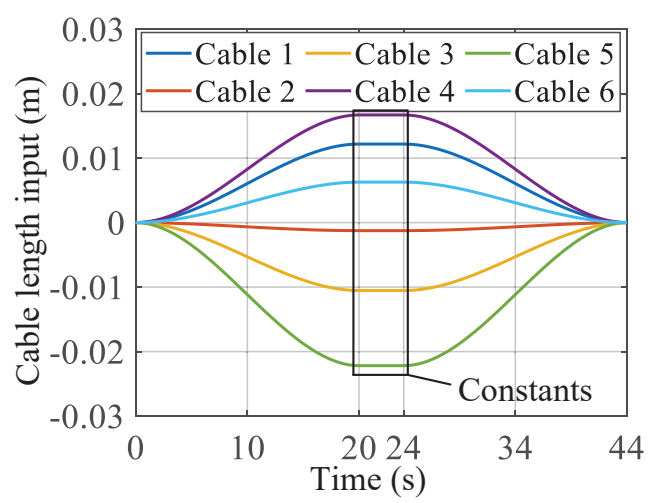

Fig. 15 Input lengths of driving cables by a pure kinematical planning method without cable deformations

Four cases of motion are studied to reveal the effect$\mathrm{s}$ of friction on the dynamical behaviors of the cabledriven continuum manipulator; those are without friction on all cables, with friction on all cables, with friction only on driving cables, and with friction only on connecting cables.

Case 1: The continuum manipulator without friction on all cables

Fig. 16 presents the joint angles of the continuum manipulator without friction on all cables. The numerical results show that if all frictions on the cables are zero, the deformations of the cables can then be ignored. Thus, the joint angles in each segment are the same, i.e., the manipulator exhibits constant curvature bending in each segment. The numerical results are identical with the planned motions denoted by the green circles in the figure.

Case 2: The continuum manipulator with friction on all cables

The friction in real mechanical systems cannot be ignored, however. Thus, a more realistic simulation is conducted in this case, that all frictions, including driving cable-hole frictions and large "S" connecting cableconduit frictions, are considered. To verify the simulation results, an experimental apparatus is designed. As shown in Fig. 17, the cable-driven continuum manipu- 


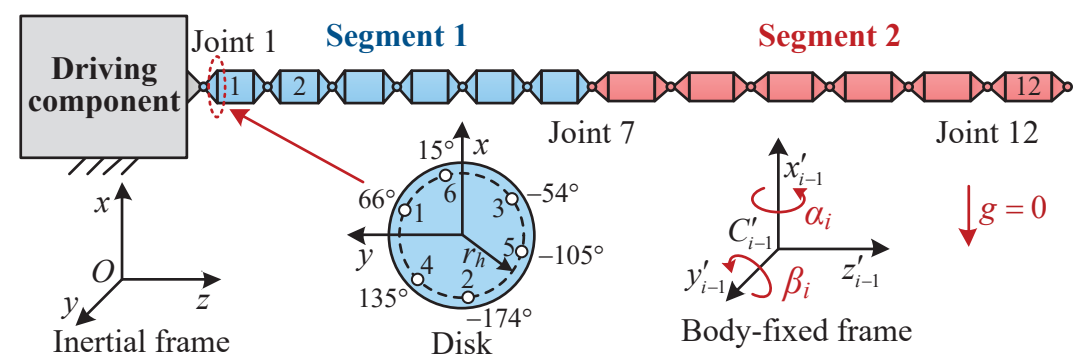

Fig. 14 A cable-driven continuum manipulator with two segments of cable-constrained synchronous rotating mechanisms

Table 1 Parameters of the cable-driven continuum manipulator with two segments of CCSRM

\begin{tabular}{|c|c|c|c|}
\hline Category & \multicolumn{2}{|c|}{ Parameter } & Value \\
\hline $\begin{array}{l}\text { Driving } \\
\text { cable }\end{array}$ & \multicolumn{2}{|c|}{$\begin{array}{l}\text { Young's modulus } \\
\text { Cross sectional area } \\
\text { Density of cable } \\
\text { Friction coefficients (if exists) }\end{array}$} & $\begin{array}{l}E=2.6 \times 10^{10} \mathrm{~Pa} \\
A=1.3273 \times 10^{-6} \mathrm{~m}^{2} \\
\rho=7.85 \times 10^{3} \mathrm{~kg} / \mathrm{m}^{3} \\
\mu=0.21 ; \mu^{\prime}=0.21\end{array}$ \\
\hline \multirow{3}{*}{$\begin{array}{l}\text { Rigid } \\
\text { link }\end{array}$} & \multicolumn{2}{|c|}{ Disk distance in the joints } & $d=0.016 \mathrm{~m}$ \\
\hline & Seg. 1 & $\begin{array}{l}\text { Mass of each link } \\
\text { Inertia matrix of each link } \\
\text { Length of each link } \\
\text { Radius of hole distributed circle }\end{array}$ & $\begin{array}{l}m_{i}=0.095 \mathrm{~kg} \\
\boldsymbol{J}^{\prime}{ }_{i}=\operatorname{diag}(66,62,19) \times 10^{-6} \mathrm{~kg} \cdot \mathrm{m}^{2} \\
L_{i}=0.085 \mathrm{~m} \\
r_{h}=0.021 \mathrm{~m}\end{array}$ \\
\hline & Seg. 2 & $\begin{array}{l}\text { Mass of each link } \\
\text { Inertia matrix of each link } \\
\text { Length of each link } \\
\text { Radius of hole distributed circle }\end{array}$ & $\begin{array}{l}m_{i}=0.070 \mathrm{~kg} \\
\boldsymbol{J}^{\prime}{ }_{i}=\operatorname{diag}(54,53,7) \times 10^{-6} \mathrm{~kg} \cdot \mathrm{m}^{2} \\
L_{i}=0.100 \mathrm{~m} \\
r_{h}=0.016 \mathrm{~m}\end{array}$ \\
\hline \multirow{3}{*}{$\begin{array}{l}\text { Connecting } \\
\text { cable }\end{array}$} & \multicolumn{2}{|c|}{$\begin{array}{l}\text { Young's modulus } \\
\text { Cross-sectional area } \\
\text { Pre-tensioning force } \\
\text { Friction coefficients (if exists) }\end{array}$} & $\begin{array}{l}E_{c}=2.6 \times 10^{10} \mathrm{~Pa} \\
A_{c}=1.3273 \times 10^{-6} \mathrm{~m}^{2} \\
T_{p}=50 \mathrm{~N} \\
\mu_{c}=0.21 ; \mu_{c}^{\prime}=0.21\end{array}$ \\
\hline & Seg. 1 & $\begin{array}{l}\text { Length of cable } \\
\text { Radius of pulley } \\
\text { Special wrap angle }\end{array}$ & $\begin{array}{l}l_{s c}=0.084 \mathrm{~m} ; l_{l c}=0.109 \mathrm{~m} \\
r_{s c}=0.006 \mathrm{~m} ; r_{l c}=0.016 \mathrm{~m} \\
\Theta_{l c}=184^{\circ}\end{array}$ \\
\hline & Seg. 2 & $\begin{array}{l}\text { Length of cable } \\
\text { Radius of pulley } \\
\text { Special wrap angle }\end{array}$ & $\begin{array}{l}l_{s c}=0.100 \mathrm{~m} ; l_{l c}=0.120 \mathrm{~m} \\
r_{s c}=0.006 \mathrm{~m} ; r_{l c}=0.016 \mathrm{~m} \\
\Theta_{l c}=158^{\circ}\end{array}$ \\
\hline $\begin{array}{l}\text { Simulation } \\
\text { parameters }\end{array}$ & $\begin{array}{l}\text { Baum } \\
\text { Model } \\
\text { Veloci }\end{array}$ & $\begin{array}{l}\text { arte parameters } \\
\text { smoothing step } \\
\text { tolerance }\end{array}$ & $\begin{array}{l}\alpha_{B}=100 ; \beta_{B}=100 \\
h=0.01 \mathrm{~s} \\
v_{\varepsilon}=1 \times 10^{-6} \mathrm{~m} / \mathrm{s} ; \\
v_{s}=1 \times 10^{-4} \mathrm{~m} / \mathrm{s} ; v_{d}=2 \times 10^{-4} \mathrm{~m} / \mathrm{s}\end{array}$ \\
\hline
\end{tabular}

lator with two segments of CCSRM is moving on an air-floating platform. Four motion capturing cameras are fixed around to record the motions of the markers installed on the rigid links. Thus, each joint angle in $x_{i-1}^{\prime}$ direction can be obtained.

Fig. 18 presents the joint angles of the continuum manipulator with friction on all cables, which indicates that the numerical results are consistent with the experiment. Due to the effects of friction, the constant curvature in each segment does not remain. In each segment, the amplitudes of joint angles roughly decrease from the root to the end except the last joint. Besides, an interesting phenomenon can be noticed that although the connecting cables of large "S" mechanisms are subjected to the kinetic frictions from the pulleys and conduits, the performance of large "S" synchronous rotating mechanisms is better than that of small " $\mathrm{S}$ " mechanisms. The reason is that the connecting stiffness of large "S" mechanisms is much larger than that of small "S" mechanisms. Thus, it may be an optimization direction of mechanical design for our next generation manipulator to keep the connecting stiff parameters of 


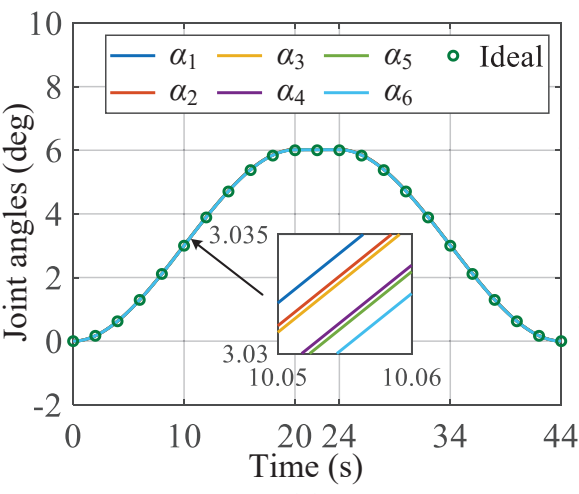

(a)

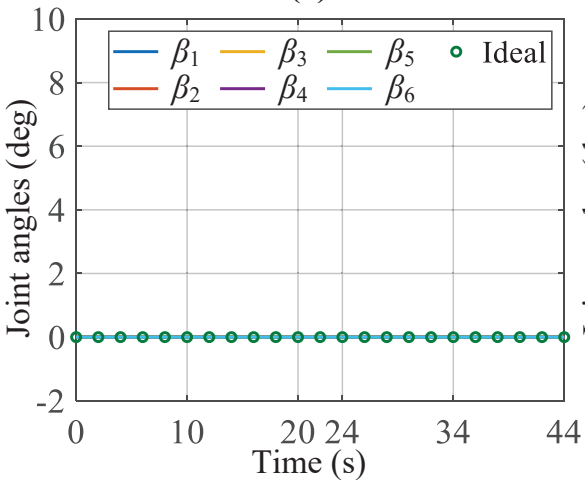

(c)

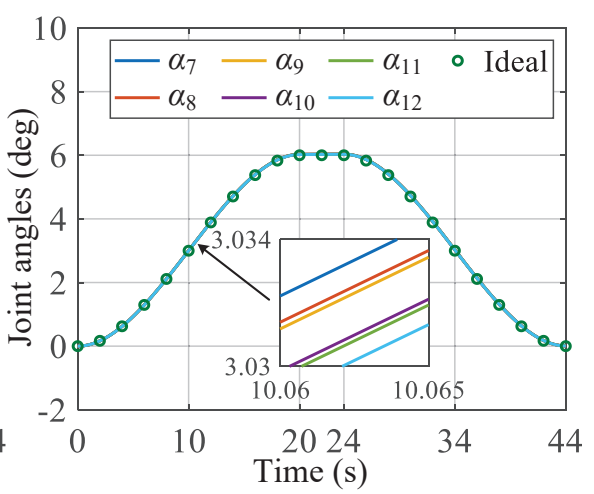

(b)

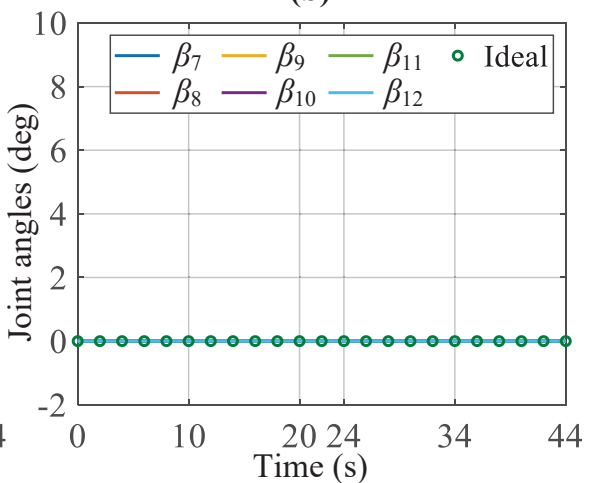

(d)

Fig. 16 Joint angles of the manipulator without friction on all cables

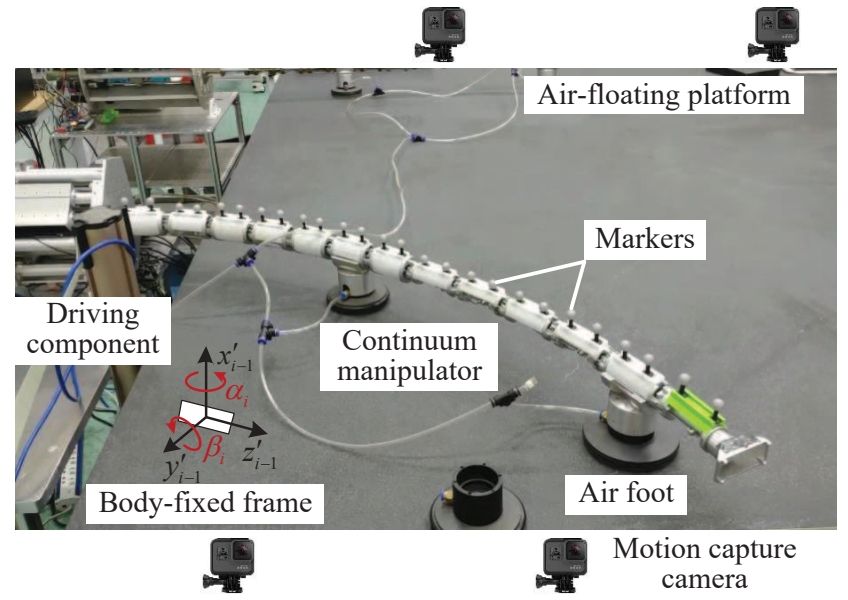

Fig. 17 Experimental apparatus to record the motion of the continuum manipulator

large and small "S" mechanisms matching. An opposite direction of motion has also been made, i.e., the planned motion is that each joint rotates $-6^{\circ}$ around $x_{i-1}^{\prime}$ axis, and then rotates back. The numerical and experimental results are illustrated in Fig. 19. From Figs. 18 and 19, it can be concluded that there is no difference between the two directions of motion. The following two cases present the effects of driving or connecting cable friction alone on the non-constant curvature of manipulator.
Case 3: The continuum manipulator with friction only on driving cables

Fig. 20 illustrates the joint angles of the manipulator with friction only on driving cables. During a time from 20 to 24 seconds, the driving cables are subjected to static frictional forces keeping the joint angles unchanged, which demonstrates that the proposed dynamic modeling method for driving cables can capture stick-slip motions well. Without the static friction, the joint angles of a segment would become to an equal value. The numerical results indicate that it is the driving cable friction which makes the joint angle amplitudes decrease from the root to the end. Another phenomenon can be noticed that the performance of the synchronous rotating mechanisms of segment 2 is better than that of segment 1 . This is because segment 1 is subjected to frictions of six driving cables, while segment 2 only suffers from frictions of three driving cables. Besides, the diameter of rigid links in segment 2 is smaller than that of segment 1, which causes the arm of frictional force smaller than that of segment 1. Due to the friction from the driving cables, a little rotation of each joint around $y_{i-1}^{\prime}$ axis has also been aroused, as shown in Fig. 20 (c) and $(\mathrm{d})$.

Case 4: The continuum manipulator with friction only on connecting cables 


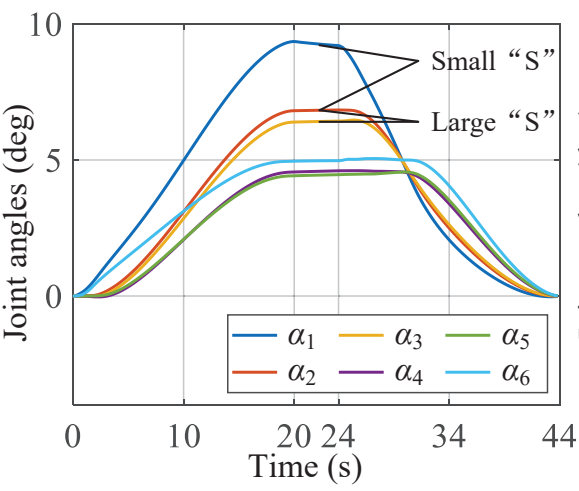

(a)

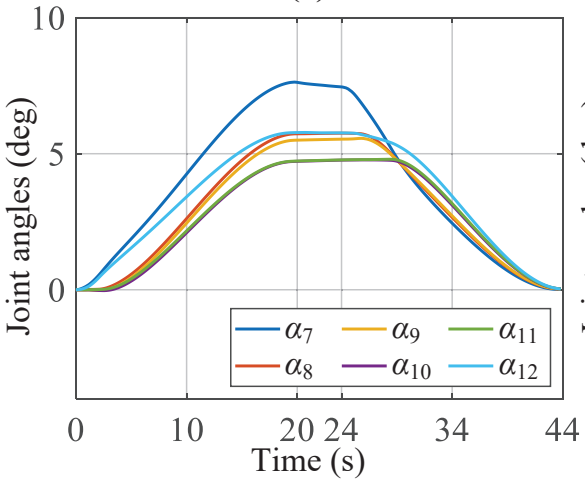

(c)

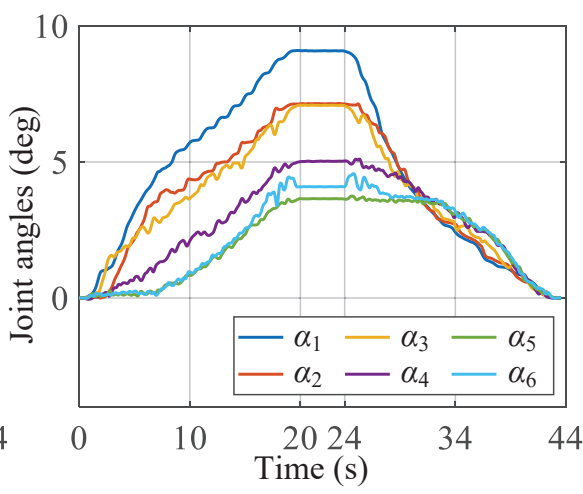

(b)

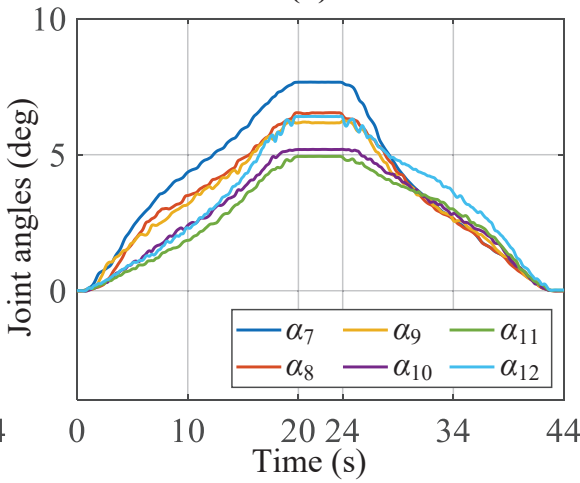

(d)

Fig. 18 Joint angles of the manipulator with friction on all cables: (a) and (c) Simulation results; (b) and (d) Experimental results

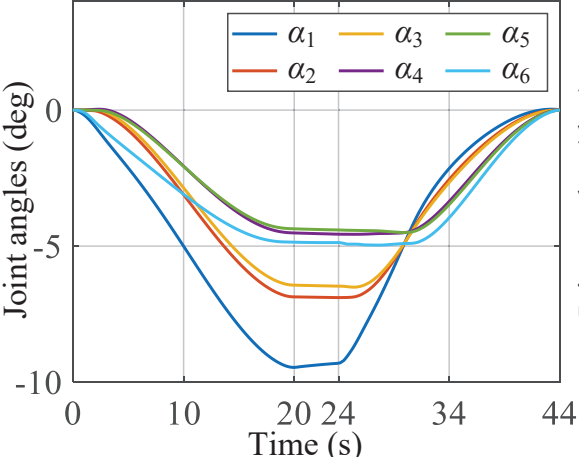

(a)

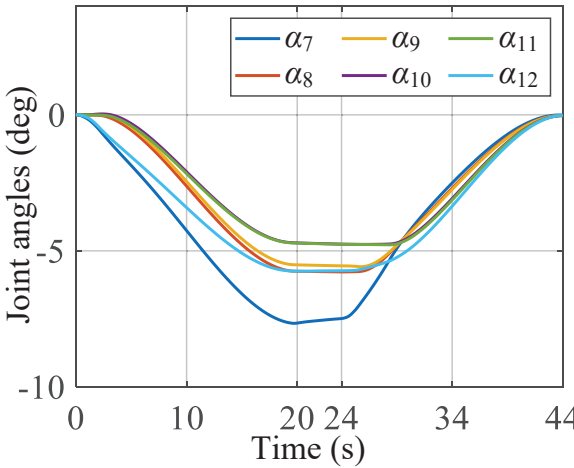

(c)

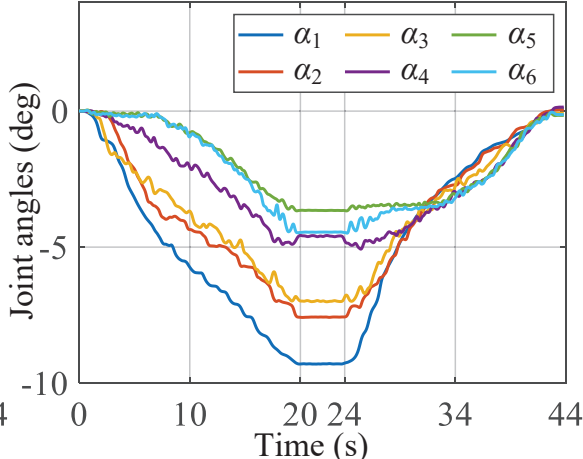

(b)

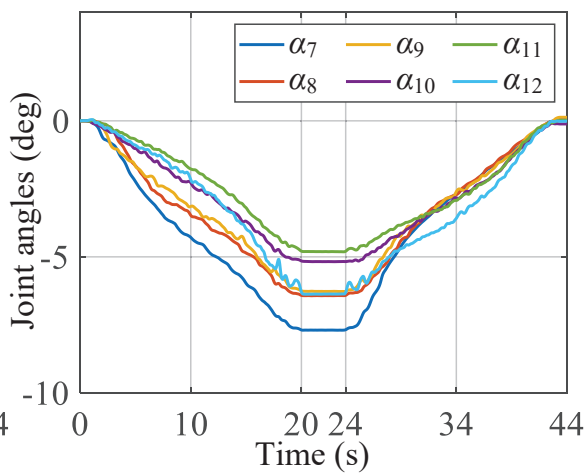

(d)

Fig. 19 Joint angles of the manipulator with friction on all cables (moving to the opposide direction): (a) and (c) Simulation results; (b) and (d) Experimental results 


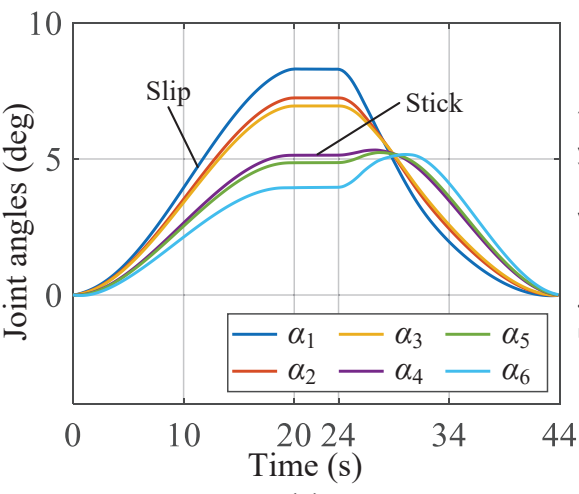

(a)

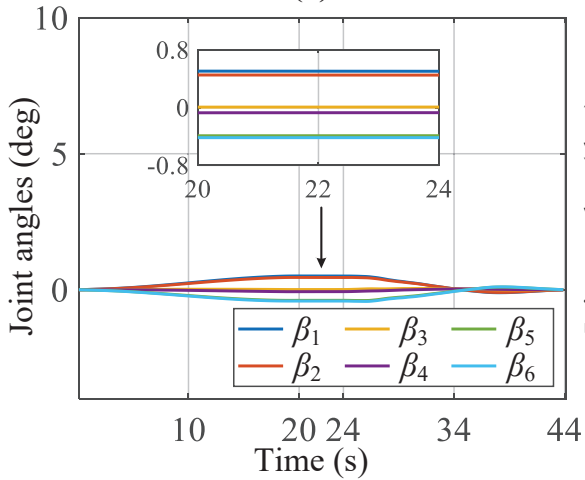

(c)

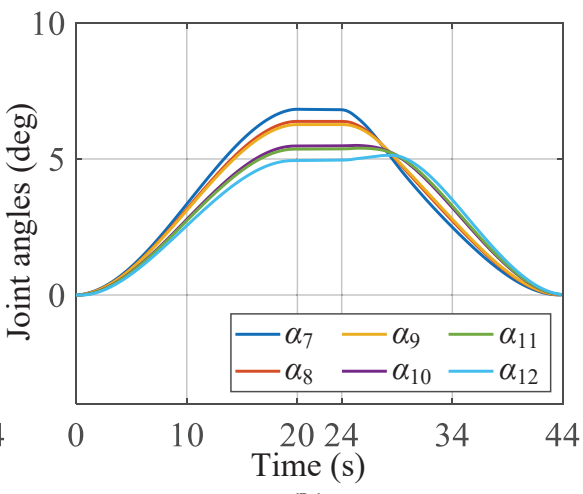

(b)

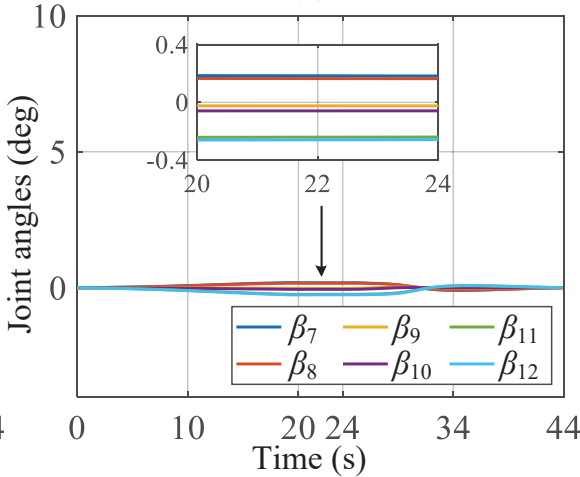

(d)

Fig. 20 Joint angles of the manipulator with friction only on driving cables

Fig. 21 presents the joint angles of the manipulator with friction acting only on connecting cables. The numerical results show that there is no difference between the two segments, and they all rotate from the two ends of the segments, while the two middle joints of the segments move last. Because of the friction from the connecting cables, small rotations around $y_{i-1}^{\prime}$ axes have also been aroused, as illustrated in Fig. 21 (c) and (d).

Fig. 22 further compares the input cable tensions for the four cases, which implies that more friction factors considered, larger amplitudes of input cable tensions needed. Besides, the amplitudes of input cable tensions for case 3 bigger than case 4, which indicates that the frictions on driving cables play a more important role in the non-constant curvature bending of the manipulator than the connecting cable frictions, which can also be concluded by comparing Figs. 20 and 21.

\section{Conclusions}

This paper presents a dynamic modeling method for a cable-driven continuum manipulator with cable-constrained synchronous rotating mechanisms. First, the driving cables are modeled based on the ALE formulation and the strategies for detecting stick-slip motions are proposed by using a trial-and-error algorithm. The stiff problems of the dynamic equations are released by a model smoothing method. Second, the dynamic modeling method for rigid links is presented by using quaternion parameters, which have the advantages of avoiding singularities and reducing the calculation of trigonometric functions to improve the calculation efficiency. Third, the connecting cables are modeled by torsional spring-dampers to avoid the further use of ALE cable elements to reduce the DOFs of whole multibody system. The frictions between the connecting cables and the conduits are considered based on a modified Coulomb friction model. At last, the numerical results are presented and verified by comparing with experiment results.

The numerical and experimental results show that if all frictions are not considered, the results are identical with planned motions, and the manipulator exhibits constant curvature bending in each segment. However, in real mechanical systems, the frictions cannot be ignored and the constant curvature bending does not remain. In each segment, the amplitudes of joint angles roughly decrease from the root to the end. Besides, the performance of the first segment of synchronous rotating mechanisms is better than that of the second segment, which is because the first segment suffers from 


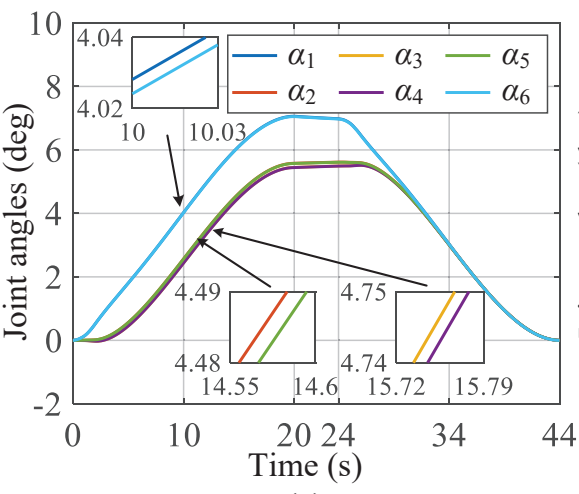

(a)

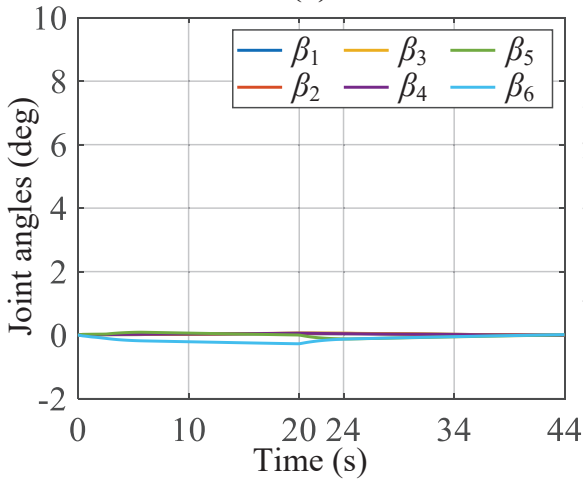

(c)

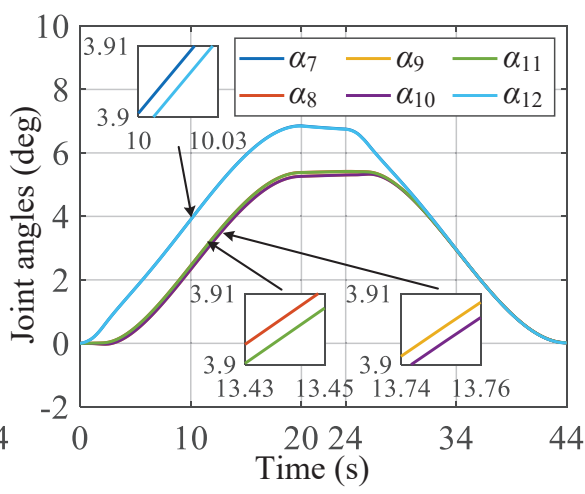

(b)

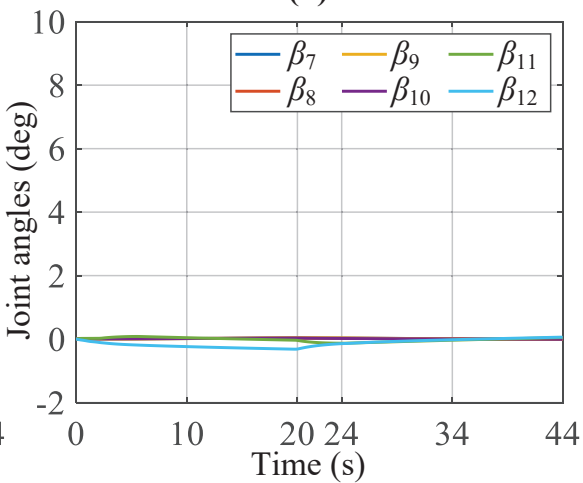

(d)

Fig. 21 Joint angles of the manipulator with friction only on connecting cables

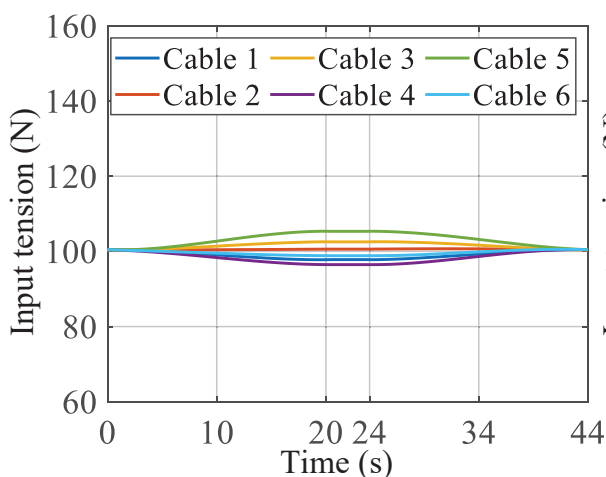

(a)

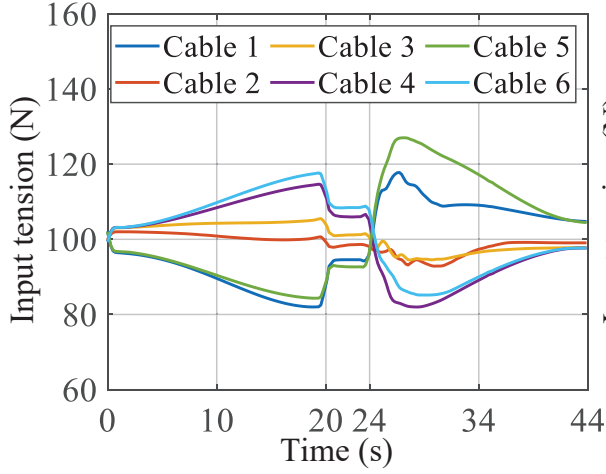

(c)

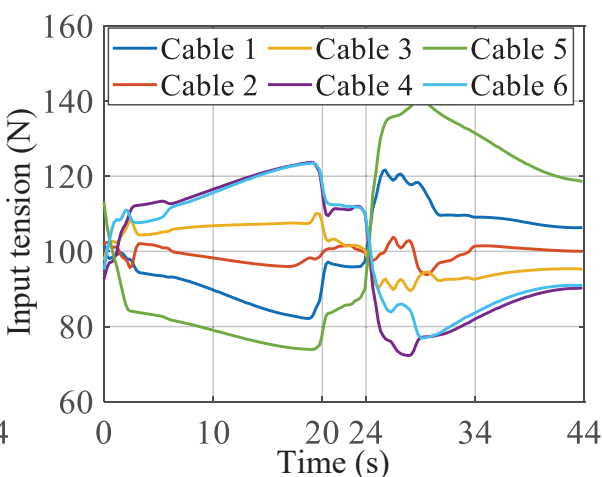

(b)

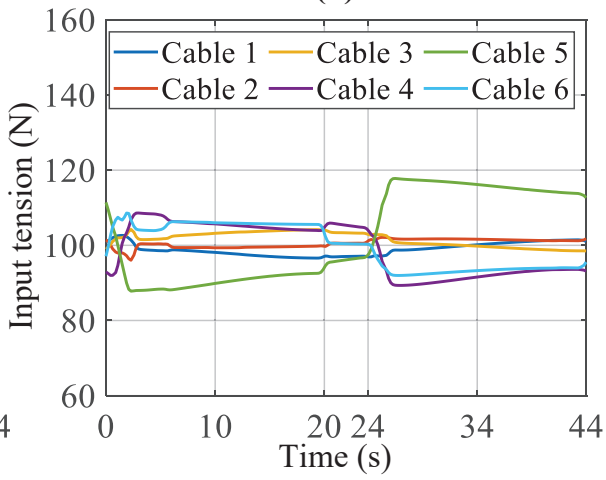

(d)

Fig. 22 Input cable tensions for different cases: (a) Case 1; (b) Case 2; (c) Case 3; (d) Case 4 
more driving cable frictions than the second segment. An interesting phenomenon can also be noticed that the performance of large "S" mechanisms is better than small "S" mechanisms. The reason is that the connecting stiffness parameter of large "S" mechanisms is larger than small "S" mechanisms. To ensure these values matching may be an optimization design direction for our next generation manipulator.

Acknowledgements This work is supported by the grants from the National Natural Science Foundation of China (Grant No. U1813216) and the Open Project of Shenzhen Institute of Artificial Intelligence and Robotics for Society (Grant No. AC01202005004).

\section{Conflict of interest}

The authors declare that they have no conflict of interest.

\section{Data availability statement}

The datasets generated during and/or analyzed during the current study are available from the corresponding author on reasonable request.

\section{References}

1. Bajo, A., Simaan, N.: Hybrid motion/force control of multi-backbone continuum robots. The International Journal of Robotics Research 35(4), 422-434 (2015)

2. Baumgarte, J.: Stabilization of constraints and integrals of motion in dynamical systems. Comput. Meth. Appl. Mech. Eng. 1(1), 1-16 (1972)

3. Bogue, R.: Robots in the nuclear industry: a review of technologies and applications. Ind. Robot 38(2), 113118 (2011)

4. Burgner-Kahrs, J., Rucker, D.C., Choset, H.: Continuum robots for medical applications: A survey. IEEE Trans. Robot. 31(6), 1261-1280 (2015)

5. Dong, X., Axinte, D., Palmer, D., Cobos, S., Raffles, M., Rabani, A., Kell, J.: Development of a slender continuum robotic system for on-wing inspection/repair of gas turbine engines. Robot. Comput.-Integr. Manuf. 44, 218$229(2017)$

6. Falkenhahn, V., Hildebrandt, A., Neumann, R., Sawodny, O.: Dynamic control of the bionic handling assistant. IEEE-ASME Trans. Mechatron. 22(1), 6-17 (2017)

7. Flores, P., MacHado, M., Seabra, E., Tavares Da Silva, M.: A parametric study on the baumgarte stabilization method for forward dynamics of constrained multibody systems. J. Comput. Nonlinear Dyn. 6(1) (2010). DOI $10.1115 / 1.4002338$

8. Huang, S., Meng, D., She, Y., Wang, X., Liang, B., Yuan, B.: Statics of continuum space manipulators with nonconstant curvature via pseudorigid-body $3 \mathrm{R}$ model. IEEE Access 6, 70854-70865 (2018)
9. Jing, Z., Xu, Q., Huang, J.: A review on kinematic analysis and dynamic stable control of space flexible manipulators. Aerospace Systems 2(1), 1-14 (2019)

10. Kan, Z., Li, F., Peng, H., Chen, B., Song, X.: Sliding cable modeling: A nonlinear complementarity function based framework. Mech. Syst. Signal Proc. 146, 107021 (2021)

11. Kan, Z., Peng, H., Chen, B.: A simple linear complementarity approach for sliding cable modeling considering friction. Mech. Syst. Signal Proc. 130, 293-314 (2019)

12. Lakhal, O., Melingui, A., Merzouki, R.: Hybrid approach for modeling and solving of kinematics of a compact bionic handling assistant manipulator. IEEE-ASME Trans. Mechatron. 21(3), 1326-1335 (2016)

13. Li, Y., Liu, Y., Meng, D., Wang, X., Liang, B.: Modeling and experimental verification of a cable-constrained synchronous rotating mechanism considering friction effect. IEEE Robot. Autom. Lett. 5(4), 5464-5471 (2020)

14. Li, Z., Wu, L., Ren, H., Yu, H.: Kinematic comparison of surgical tendon-driven manipulators and concentric tube manipulators. Mech. Mach. Theory 107, 148-165 (2017)

15. Liu, T., Mu, Z., Wang, H., Xu, W., Li, Y.: A cable-driven redundant spatial manipulator with improved stiffness and load capacity. In: 2018 IEEE/RSJ International Conference on Intelligent Robots and Systems (IROS), pp. $6628-6633$

16. Liu, T., Xu, W., Yang, T., Li, Y.: A hybrid active and passive cable-driven segmented redundant manipulator design, kinematics and planning. IEEE-ASME Trans. Mechatron. 26(2), 930-942 (2021)

17. Liu, Z., Zhang, X., Cai, Z., Peng, H., Wu, Z.: Real-time dynamics of cable-driven continuum robots considering the cable constraint and friction effect. IEEE Robot. Autom. Lett. pp. 1-1 (2021). DOI 10.1109/LRA.2021. 3086413

18. Ma, S., Liang, B., Wang, T.: Dynamic analysis of a hyperredundant space manipulator with a complex rope network. Aerosp. Sci. Technol. 100, 105768 (2020)

19. Peng, J., Xu, W., Liu, T., Yuan, H., Liang, B.: Endeffector pose and arm-shape synchronous planning methods of a hyper-redundant manipulator for spacecraft repairing. Mech. Mach. Theory 155, 104062 (2021)

20. Peng, J., Xu, W., Yang, T., Hu, Z., Liang, B.: Dynamic modeling and trajectory tracking control method of segmented linkage cable-driven hyper-redundant robot. Nonlinear Dyn. 101(1), 233-253 (2020)

21. Peng, Y., Wei, Y., Zhou, M.: Efficient modeling of cable-pulley system with friction based on arbitrarylagrangian-eulerian approach. Appl. Math. Mech.-Engl. Ed. 38(12), 1785-1802 (2017)

22. Pennestrì, E., Rossi, V., Salvini, P., Valentini, P.P.: Review and comparison of dry friction force models. Nonlinear Dyn. 83(4), 1785-1801 (2016)

23. Qi, P., Liu, C., Ataka, A., Lam, H.K., Althoefer, K.: Kinematic control of continuum manipulators using a fuzzymodel-based approach. IEEE Trans. Ind. Electron. 63(8), 5022-5035 (2016)

24. Roesthuis, R.J., Misra, S.: Steering of multisegment continuum manipulators using rigid-link modeling and FBGbased shape sensing. IEEE Trans. Robot. 32(2), 372-382 (2016)

25. Rone, W.S., Ben-Tzvi, P.: Continuum robot dynamic$\mathrm{s}$ utilizing the principle of virtual power. IEEE Trans. Robot. 30(1), 275-287 (2014)

26. Rong, B., Rui, X., Tao, L., Wang, G.: Theoretical modeling and numerical solution methods for flexible multibody system dynamics. Nonlinear Dyn. 98(2), 1519-1553 (2019) 
27. Song, N., Peng, H., Kan, Z., Chen, B.: A novel nonsmooth approach for flexible multibody systems with contact and friction in 3d space. Nonlinear Dyn. 102(3), 1375-1408 (2020)

28. Tonapi, M.M., Godage, I.S., Vijaykumar, A.M., Walker, I.D.: A novel continuum robotic cable aimed at applications in space. Adv. Robot. 29(13), 861-875 (2015)

29. Wang, Z., Tian, Q., Hu, H., Flores, P.: Nonlinear dynamics and chaotic control of a flexible multibody system with uncertain joint clearance. Nonlinear Dyn. 86(3), 1571-1597 (2016)

30. Xiang, W., Yan, S., Wu, J.: Dynamic analysis of planar mechanical systems considering stick-slip and stribeck effect in revolute clearance joints. Nonlinear Dyn. 95(1), 321-341 (2019)

31. Xu, W., Mu, Z., Liu, T., Liang, B.: A modified modal method for solving the mission-oriented inverse kinematics of hyper-redundant space manipulators for on-orbit servicing. Acta Astronaut. 139, 54-66 (2017)

32. Xu, X., Zhong, W.: On the numerical influences of inertia representation for rigid body dynamics in terms of unit quaternion. J. Appl. Mech.-Trans. ASME 83(6) (2016). DOI 10.1115/1.4033031

33. Yang, C., Geng, S., Walker, I., Branson, D.T., Liu, J., Dai, J.S., Kang, R.: Geometric constraint-based modeling and analysis of a novel continuum robot with shape memory alloy initiated variable stiffness. The International Journal of Robotics Research 39(14), 1620-1634 (2020)

34. Yu, L., Zhao, Z., Tang, J., Ren, G.: Integration of absolute nodal elements into multibody system. Nonlinear Dyn. 62(4), 931-943 (2010)

35. Yuan, H., Zhou, L., Xu, W.: A comprehensive static model of cable-driven multi-section continuum robots considering friction effect. Mech. Mach. Theory 135, 130-149 (2019)

36. Zhang, R., Yu, Y., Wang, Q., Wang, Q.: An improved implicit method for mechanical systems with set-valued friction. Multibody Syst. Dyn. 48(2), 211-238 (2020)

37. Zhang, X., Qi, Z., Wang, G., Guo, S.: Model smoothing method of contact-impact dynamics in flexible multibody systems. Mech. Mach. Theory 138, 124-148 (2019)

38. Zhao, Z., Lu, J., Wang, Q., Liu, C., Wang, Q.: The effect of non-spherical aspect of a dimer on the dynamic behaviors. Nonlinear Dyn. 94(3), 2191-2204 (2018)

39. Zheng, X., Li, J., Wang, Q., Liao, Q.: A methodology for modeling and simulating frictional translational clearance joint in multibody systems including a flexible slider part. Mech. Mach. Theory 142, 103603 (2019)

40. Zheng, X., Zhang, F., Wang, Q.: Modeling and simulation of planar multibody systems with revolute clearance joints considering stiction based on an LCP method. Mech. Mach. Theory 130, 184-202 (2018) 